\title{
Commutators of Multilinear Calderón-Zygmund Operator and BMO Functions in Herz-Morrey Spaces with Variable Exponents
}

\author{
Canqin Tang, ${ }^{1}$ Qing $\mathrm{Wu},{ }^{1}$ and Jingshi $X \mathrm{u}^{2}$ \\ ${ }^{1}$ Department of Mathematics, Dalian Maritime University, Dalian 116026, China \\ ${ }^{2}$ Department of Mathematics, Hainan Normal University, Haikou 571158, China
}

Correspondence should be addressed to Jingshi Xu; jingshixu@126.com

Received 25 December 2013; Accepted 25 April 2014; Published 25 May 2014

Academic Editor: Yongsheng S. Han

Copyright (c) 2014 Canqin Tang et al. This is an open access article distributed under the Creative Commons Attribution License, which permits unrestricted use, distribution, and reproduction in any medium, provided the original work is properly cited.

We obtain the boundedness of a commutator generated by multilinear Calderón-Zygmund operator and BMO functions in HerzMorrey spaces with variable exponents.

\section{Introduction}

In recent decades, variable exponent function spaces have attracted much attention. Since Kováčik and Rákosník [1] clarified fundamental properties of the variable Lebesgue and Sobolev spaces, there are many spaces studied, such as Besov and Trieble-Lizorkin spaces with variable exponents, Hardy spaces with variable exponents, Bessel potential spaces with a variable exponent, and Herz-Morrey spaces with variable exponents; see [2-13]. Recently, multilinear operators and their commutators in variable exponent function spaces are also intensively studied by a significant number of authors, such as multilinear commutators of multilinear singular integral with Lipschitz functions and $\mathrm{BMO}$ functions, respectively, in [14-19], multilinear commutators of BMO functions and multilinear singular integral operators with nonsmooth kernels in [20,21], a vector-estimate of higher order commutators on Herz-Morrey spaces with variable exponent in [22], maximal multilinear commutators and maximal iterated commutators generated by multilinear operators and Lipschitz functions in [23], and weighted estimates for vectorvalued commutators of multilinear operators in [24].

Motivated by the above results, in this paper, we will consider the boundedness of a commutator generated by a multilinear Calderón-Zygmund operator and BMO functions on the variable Herz-Morrey spaces.

To state the main result of this paper, we need to recall more notations.
Let $T$ be a multilinear singular integral operator which is initially defined on the $m$-fold product of the Schwartz space $\delta\left(\mathbb{R}^{n}\right)$. Its values are taken in the space of tempered distributions $S^{\prime}\left(\mathbb{R}^{n}\right)$ such that, for $x \notin \cap_{j=1}^{m} \operatorname{supp} f_{j}, \vec{f}=$ $\left(f_{1}, \ldots, f_{m}\right)$,

$$
T \vec{f}(x)=\int_{\left(\mathbb{R}^{n}\right)^{m}} K\left(x, y_{1}, \ldots, y_{m}\right) \prod_{i=1}^{m} f_{i}\left(y_{i}\right) d y_{1} \cdots d y_{m},
$$

where $f_{1}, \ldots, f_{m} \in L_{C}^{\infty}\left(\mathbb{R}^{n}\right)$ (the space of compactly supported bounded functions). Here, the kernel $K$ is a function in $\left(\mathbb{R}^{n}\right)^{m+1}$ away from the diagonal $y_{0}=y_{1}=\cdots=y_{m}$ and satisfies the standard estimates

$$
\begin{gathered}
\left|K\left(x, y_{1}, \ldots, y_{m}\right)\right| \leq A\left(\left|x-y_{1}\right|+\cdots+\left|x-y_{m}\right|\right)^{-m n}, \\
\left|K\left(x, y_{1}, \ldots, y_{m}\right)-K\left(x^{\prime}, y_{1}, \ldots, y_{m}\right)\right| \\
\leq \frac{A\left|x-x^{\prime}\right|^{\epsilon}}{\left(\left|x-y_{1}\right|+\cdots+\left|x-y_{m}\right|\right)^{m n+\epsilon}}
\end{gathered}
$$

provided that $\left|x-x^{\prime}\right| \leq(1 / 2) \max \left\{\left|x-y_{1}\right|+\cdots+\left|x-y_{m}\right|\right\}$, and, for each $1 \leq i \leq m$,

$$
\begin{aligned}
& \left|K\left(x, y_{1}, \ldots, y_{i}, \ldots, y_{m}\right)-K\left(x, y_{1}, \ldots, y_{i}^{\prime}, \ldots, y_{m}\right)\right| \\
& \quad \leq \frac{A\left|y_{i}-y_{i}^{\prime}\right|^{\epsilon}}{\left(\sum_{j=1}^{m}\left|x-y_{j}\right|\right)^{m n+\epsilon}}
\end{aligned}
$$


provided that $\left|y_{i}-y_{i}^{\prime}\right| \leq(1 / 2) \max \left\{\left|x-y_{1}\right|+\cdots+\left|x-y_{m}\right|\right\}$, where $A$ and $\epsilon$ are positive constants.

Such kernels are called the $m$-linear Calderón-Zygmund kernels and the collection of such functions is denoted by $m-\operatorname{CZK}(A, \epsilon)$ in [25].

Let $T$ be as in (1) with an $m-\operatorname{CZK}(A, \epsilon)$ kernel. If, for some $1<q_{1}, q_{2}, \ldots, q_{m}<\infty, T$ is bounded from $L^{q_{1}} \times$ $L^{q_{2}} \times \cdots \times L^{q_{m}}$ to $L^{q}$ with $1 / q_{1}+1 / q_{2}+\cdots+1 / q_{m}=1 / q$, then we say $T$ is an $m$-linear Calderón-Zygmund operator. Grafakos and Torres in [25] showed that if $T$ is an $m$-linear Calderón-Zygmund operator, then $T$ is bounded from $L^{q_{1}} \times$ $L^{q_{2}} \times \cdots \times L^{q_{m}}$ to $L^{q}$ for any $1<q_{1}, q_{2}, \ldots, q_{m}<\infty$ such that $1 / q_{1}+1 / q_{2}+\cdots+1 / q_{m}=1 / q$. Then, Grafakos and Torres in [26] obtained weighted norm inequalities for multilinear Calderón-Zygmund operators.

If $b \in L_{\text {loc }}^{1}\left(\mathbb{R}^{n}\right)$ (the set of all complex-valued locally integrable functions on $\mathbb{R}^{n}$ ), set

$$
\|b\|_{*}:=\sup _{B} \frac{1}{|B|} \int_{B}\left|b(x)-b_{B}\right| d x,
$$

where the supremum is taken over all balls in $\mathbb{R}^{n}, b_{B}$ is the mean of $b$ on $B$, and what follows $|E|$ is the Lebesgue measure of measurable set $E$ in $\mathbb{R}^{n}$. A function $b$ is called bounded mean oscillation if $\|b\|_{*}<\infty$ and $\operatorname{BMO}\left(\mathbb{R}^{n}\right)$ is the set of all locally integrable functions $b$ on $\mathbb{R}^{n}$ such that $\|b\|_{*}<\infty$.

Let $\vec{b}=\left(b_{1}, \ldots, b_{m}\right)$ and $b_{j} \in \operatorname{BMO}\left(\mathbb{R}^{n}\right)$ for $1 \leq j \leq m$. We will consider the commutator $[\vec{b}, T]$, which is defined for suitable functions $f_{1}, \ldots, f_{m}$ by

$$
[\vec{b}, T] \vec{f}(x):=\sum_{\sigma}(-1)^{\left|\sigma^{\prime}\right|} b_{\sigma}(x) T b_{\sigma^{\prime}} \vec{f}(x)
$$

where $\vec{f}=\left(f_{1}, \ldots, f_{m}\right), \sigma$ denotes a subset of $\{1,2, \ldots, m\}, \sigma^{\prime}$ is the complement of $\sigma$ in $\{1,2, \ldots, m\},\left|\sigma^{\prime}\right|$ denotes the number of elements of $\sigma^{\prime}, b_{\sigma}(x)=\prod_{j \in \sigma} b_{j}(x), b_{\sigma^{\prime}} \vec{f}=$ $\left(g_{1}, \ldots, g_{m}\right)$, when $j \in \sigma^{\prime}, g_{j}=b_{j} f_{j}$, otherwise, $g_{j}=f_{j}$.

Definition 1. Let $p(\cdot): \mathbb{R}^{n} \rightarrow[1, \infty)$ be a measurable function.

(i) The Lebesgue space with variable exponent $L^{p(\cdot)}\left(\mathbb{R}^{n}\right)$ is defined by

$$
\begin{gathered}
L^{p(\cdot)}\left(\mathbb{R}^{n}\right):=\left\{f \text { is measurable: } \int_{\mathbb{R}^{n}}\left(\frac{|f(x)|}{\lambda}\right)^{p(x)} d x<\infty\right. \\
\text { for some } \lambda>0\} .
\end{gathered}
$$

(ii) The space $L_{\text {loc }}^{p(\cdot)}\left(\mathbb{R}^{n}\right)$ is defined by

$$
\begin{aligned}
& L_{\text {loc }}^{p(\cdot)}\left(\mathbb{R}^{n}\right) \\
& :=\left\{f: f \chi_{K} \in L^{p(\cdot)}\left(\mathbb{R}^{n}\right) \text { for all compact subsets } K \subset \mathbb{R}^{n}\right\},
\end{aligned}
$$

where, and in what follows, $\chi_{S}$ denotes the characteristic function of a measurable set $S \subset \mathbb{R}^{n}$.

$L^{p(\cdot)}\left(\mathbb{R}^{n}\right)$ is a Banach function space when equipped with the norm

$$
\|f\|_{L^{p(\cdot)}}:=\inf \left\{\lambda>0: \int_{\mathbb{R}^{n}}\left(\frac{|f(x)|}{\lambda}\right)^{p(x)} d x \leq 1\right\} .
$$

Letting $p(\cdot): \mathbb{R}^{n} \rightarrow(0, \infty)$, we denote

$$
p_{-}:=\text {ess } \inf _{x \in \mathbb{R}^{n}} p(x), \quad p_{+}:=\text {ess } \inf _{x \in \mathbb{R}^{n}} p(x) .
$$

The set $\mathscr{P}\left(\mathbb{R}^{n}\right)$ consists of all $p(\cdot)$ satisfying $p_{-}>1$ and $p_{+}<\infty ; \mathscr{P}^{0}\left(\mathbb{R}^{n}\right)$ consists of all $p(\cdot)$ satisfying $p_{-}>0$ and $p_{+}<\infty . L^{p(\cdot)}$ can be similarly defined as mentioned above for $p(\cdot) \in \mathscr{P}^{0}\left(\mathbb{R}^{n}\right) \cdot p^{\prime}(\cdot)$ means the conjugate exponent of $p(\cdot)$ that means $1 / p(\cdot)+1 / p^{\prime}(\cdot)=1$.

Let $f \in L_{\text {loc }}^{1}\left(\mathbb{R}^{n}\right)$. Then the standard Hardy-Littlewood maximal function of $f$ is defined by

$$
M f(x)=\sup _{x \in B} \frac{1}{|B|} \int_{B}|f(y)| d y, \quad \forall x \in \mathbb{R}^{n},
$$

where $B$ is a ball and $|B|$ is the volume of $B$. Let $\mathscr{B}\left(\mathbb{R}^{n}\right)$ be the set of $p(\cdot) \in \mathscr{P}\left(\mathbb{R}^{n}\right)$ such that $M$ is bounded on $L^{p(\cdot)}\left(\mathbb{R}^{n}\right)$. For more on $\mathscr{B}\left(\mathbb{R}^{n}\right)$, see $[27,28]$.

Definition 2. We say that a function $\alpha(\cdot): \mathbb{R}^{n} \rightarrow \mathbb{R}$ is locally $\log$-Hölder continuous if there exists a constant $C$ such that

$$
\begin{array}{r}
|\alpha(x)-\alpha(y)| \leq \frac{C}{\log (e+1 /|x-y|)}, \\
x, y \in \mathbb{R}^{n}, \quad|x-y|<\frac{1}{2} .
\end{array}
$$

If

$$
|\alpha(x)-\alpha(0)| \leq \frac{C}{\log (e+1 /|x|)}, \quad \forall x \in \mathbb{R}^{n},
$$

then we say that $\alpha(\cdot)$ is log-Hölder continuous at the origin. If there exists $\alpha_{\infty} \in \mathbb{R}$ such that

$$
\left|\alpha(x)-\alpha_{\infty}\right| \leq \frac{C}{\log (e+|x|)}, \quad \forall x \in \mathbb{R}^{n},
$$

then we say that $\alpha(\cdot)$ is log-Hölder continuous at the infinity. If $\alpha(\cdot)$ is both locally log-Hölder continuous and log-Hölder continuous at the infinity, then we say $\alpha(\cdot)$ is global logHölder continuous.

We denote by $\mathscr{P}_{0}^{\log }\left(\mathbb{R}^{n}\right)$ and $\mathscr{P}_{\infty}^{\log }\left(\mathbb{R}^{n}\right)$ the class of all exponents $p \in \mathscr{P}\left(\mathbb{R}^{n}\right)$ which is log-Hölder continuous at the origin and at the infinity, respectively.

Next, we recall the definitions of Herz spaces and HerzMorrey spaces with variable exponents. We use the following notations. For each $k \in \mathbb{Z}$, we define

$$
\begin{gathered}
B_{k}:=\left\{x \in \mathbb{R}^{n}:|x| \leq 2^{k}\right\}, \\
D_{k}:=B_{k} \backslash B_{k-1}, \quad \chi_{k}:=\chi_{D_{k}} .
\end{gathered}
$$


Definition 3 (see [29, Definition 2]). Let $q \in(0, \infty], p(\cdot) \in$ $\mathscr{P}\left(\mathbb{R}^{n}\right)$, and $\alpha(\cdot): \mathbb{R}^{n} \rightarrow \mathbb{R}$ with $\alpha \in L^{\infty}\left(\mathbb{R}^{n}\right)$. The homogeneous Herz space $\dot{K}_{q, p(\cdot)}^{\alpha(\cdot)}\left(\mathbb{R}^{n}\right)$ is defined as the set of all $f \in L_{\text {loc }}^{p(\cdot)}\left(\mathbb{R}^{n} \backslash\{0\}\right)$ such that

$$
\|f\|_{\dot{K}_{q, p(\cdot)}^{\alpha(\cdot)}}:=\left\{\sum_{k \in \mathbb{Z}}\left\|2^{k \alpha(\cdot)} f \chi_{k}\right\|_{p(\cdot)}^{q}\right\}^{1 / q}<\infty,
$$

with the usual modifications when $q=\infty$.

Definition 4 (see [30, Definition 2]). Let $0<q<\infty, p(\cdot) \in$ $\mathscr{P}\left(\mathbb{R}^{n}\right), 0 \leq \lambda<\infty$ and $\alpha(\cdot): \mathbb{R}^{n} \rightarrow \mathbb{R}$ with $\alpha \in L^{\infty}\left(\mathbb{R}^{n}\right)$. The Herz-Morrey space $M \dot{K}_{q, p(\cdot)}^{\alpha(\cdot), \lambda}\left(\mathbb{R}^{n}\right)$ with variable exponents is defined by

$$
M \dot{K}_{q, p(\cdot)}^{\alpha(\cdot), \lambda}\left(\mathbb{R}^{n}\right):=\left\{f \in L_{\text {loc }}^{p(\cdot)}\left(\mathbb{R}^{n} \backslash\{0\}\right):\|f\|_{M \dot{K}_{q, p(\cdot)}^{\alpha(\cdot), \lambda}\left(\mathbb{R}^{n}\right)}<\infty\right\},
$$

where

$$
\|f\|_{M \dot{K}_{q, p(\cdot)}^{\alpha(\cdot), \lambda}\left(\mathbb{R}^{n}\right)}:=\sup _{L \in \mathbb{Z}} 2^{-L \lambda}\left(\sum_{k=-\infty}^{L}\left\|2^{k \alpha(\cdot)} f \chi_{k}\right\|_{L^{p(\cdot)}\left(\mathbb{R}^{n}\right)}^{q}\right)^{1 / q} .
$$

If $\alpha(\cdot)$ is a constant, then $M \dot{K}_{q, p(\cdot)}^{\alpha(\cdot), \lambda}\left(\mathbb{R}^{n}\right)=M \dot{K}_{q, p(\cdot)}^{\alpha, \lambda}\left(\mathbb{R}^{n}\right)$ was defined in [30]. If $\lambda=0$, then $M \dot{K}_{q, p(\cdot)}^{\alpha(\cdot), \lambda}\left(\mathbb{R}^{n}\right)=$ $\dot{K}_{q, p(\cdot)}^{\alpha(\cdot)}\left(\mathbb{R}^{n}\right)$. If both $\alpha(\cdot)$ and $p(\cdot)$ are constant and $\lambda=0$, then $M \dot{K}_{q, p(\cdot)}^{\alpha(\cdot), \lambda}\left(\mathbb{R}^{n}\right)=\dot{K}_{q, p}^{\alpha}\left(\mathbb{R}^{n}\right)$ is the classical Herz space in [31].

Lemma 5 (see $[30$, Lemma 1 and $(10)]$ ). If $p(\cdot) \in \mathscr{B}\left(\mathbb{R}^{n}\right)$, then there exist constants $\delta_{1}, \delta_{2} \in(0,1)$ and $C>0$ such that, for all balls $B$ in $\mathbb{R}^{n}$ and all measurable subsets $S \subset B$,

$$
\begin{aligned}
& \frac{\left\|\chi_{S}\right\|_{L^{p(\cdot)}\left(\mathbb{R}^{n}\right)}}{\left\|\chi_{B}\right\|_{L^{p \cdot()}\left(\mathbb{R}^{n}\right)}} \leq C\left(\frac{|S|}{|B|}\right)^{\delta_{1}}, \\
& \frac{\left\|\chi_{S}\right\|_{L^{p^{(\cdot)}\left(\mathbb{R}^{n}\right)}}}{\left\|\chi_{B}\right\|_{L^{p^{\prime} \cdot(\cdot)}\left(\mathbb{R}^{n}\right)}} \leq C\left(\frac{|S|}{|B|}\right)^{\delta_{2}} .
\end{aligned}
$$

There is a position to state our result.

Theorem 6. Let $b_{i} \in B M O\left(\mathbb{R}^{n}\right)$ and $p(\cdot), p_{i}(\cdot) \in \mathscr{B}\left(\mathbb{R}^{n}\right)$ satisfy $1 / p(x)=1 / p_{1}(x)+1 / p_{2}(x)+\cdots+1 / p_{m}(x), 1<p_{i}^{-} \leq$ $p_{i}^{+}<n / \lambda_{i},\left(p(\cdot) / p_{*}\right)^{\prime} \in \mathscr{B}\left(\mathbb{R}^{n}\right)$ for some $0<p_{*}<p_{-}$, $i=1,2, \ldots, m$. Let $0<q_{i}<\infty, 0 \leq \lambda_{i}<\infty$ and $\alpha_{i}(\cdot) \in L^{\infty}\left(\mathbb{R}^{n}\right) \cap \mathscr{P}_{0}^{\log }\left(\mathbb{R}^{n}\right) \cap \mathscr{P}_{\infty}^{\log }\left(\mathbb{R}^{n}\right)$ for $i=1,2, \ldots, m$ with

$$
\lambda_{i}-n \delta_{1}<\alpha_{i}^{-}<\alpha_{i}^{+}<n \delta_{2},
$$

where $\delta_{1}, \delta_{2} \in(0,1)$ are the constants appearing in (18). Suppose that $\lambda=\sum_{i=1}^{m} \lambda_{i}, \alpha(\cdot)=\sum_{i=1}^{m} \alpha_{i}(\cdot)$, and $1 / q=$ $\sum_{i=1}^{m}\left(1 / q_{i}\right)$. Then

$$
\|[\vec{b}, T](\vec{f})\|_{M \dot{K}_{q, p(\cdot)}^{\alpha(\cdot), \lambda}\left(\mathbb{R}^{n}\right)} \leq C \prod_{i=1}^{m}\left\|b_{i}\right\|_{*}\left\|f_{i}\right\|_{M \dot{K}_{q_{i}, p_{i}(\cdot)}^{\alpha_{i}(\cdot), \lambda_{i}}\left(\mathbb{R}^{n}\right)}
$$

with the constant $C>0$ independent of $\vec{f}=\left(f_{1}, f_{2}, \ldots, f_{m}\right)$.
Remark 7. Let $\lambda_{i}=0$, and then the commutator $[\vec{b}, T]$ is bounded from the product of variable exponents Herz spaces $\dot{K}_{q_{1}, p_{1}(\cdot)}^{\alpha_{1}(\cdot)}\left(\mathbb{R}^{n}\right) \times \dot{K}_{q_{2}, p_{2}(\cdot)}^{\alpha_{2}(\cdot)}\left(\mathbb{R}^{n}\right) \times \cdots \times \dot{K}_{q_{m}, p_{m}(\cdot)}^{\alpha_{m}(\cdot)}\left(\mathbb{R}^{n}\right)$ to variable exponents Herz space $\dot{K}_{q, p(\cdot)}^{\alpha(\cdot)}\left(\mathbb{R}^{n}\right)$ when $-n \delta_{1}<\alpha_{i}^{-}<\alpha_{i}^{+}<$ $n \delta_{2}$.

Finally, we point out that $C$ denotes a positive constant which may be different at different occurrences.

\section{Proof of the Main Result}

To give our proof, we need some lemmas.

Lemma 8 (see [32, Proposition 2]). If $\alpha(\cdot) \in L^{\infty}\left(\mathbb{R}^{n}\right) \cap$ $\mathscr{P}_{0}^{\log }\left(\mathbb{R}^{n}\right) \cap \mathscr{P}_{\infty}^{\log }\left(\mathbb{R}^{n}\right), 0 \leq \lambda<\infty, p(\cdot) \in \mathscr{P}\left(\mathbb{R}^{n}\right)$, and $q \in(0, \infty)$, then

$$
\|f\|_{M \dot{K}_{q, p(\cdot)}^{\alpha(\cdot), \lambda}\left(\mathbb{R}^{n}\right)}
$$

$$
\begin{gathered}
\approx \max \left\{\sup _{L \leq 0, L \in \mathbb{Z}} 2^{-L \lambda}\left(\sum_{k=-\infty}^{L} 2^{k \alpha(0) q}\left\|f \chi_{k}\right\|_{L^{p(\cdot)}\left(\mathbb{R}^{n}\right)}^{q}\right)^{1 / q},\right. \\
\sup _{L>0, L \in \mathbb{Z}}\left[2^{-L \lambda}\left(\sum_{k=-\infty}^{-1} 2^{k \alpha(0) q}\left\|f \chi_{k}\right\|_{L^{p(\cdot)}\left(\mathbb{R}^{n}\right)}^{q}\right)^{1 / q}\right. \\
\left.\left.+2^{-L \lambda}\left(\sum_{k=0}^{L} 2^{k \alpha_{\infty} q}\left\|f \chi_{k}\right\|_{L^{p(\cdot)}\left(\mathbb{R}^{n}\right)}^{q}\right)^{1 / q}\right]\right\} .
\end{gathered}
$$

Lemma 9 (see [30, Lemma 2]). If $p(\cdot) \in \mathscr{B}\left(\mathbb{R}^{n}\right)$, then there exists a constant $C>0$ such that, for all balls $B$ in $\mathbb{R}^{n}$,

$$
C^{-1} \leq \frac{1}{|B|}\left\|\chi_{B}\right\|_{L^{p(\cdot)}\left(\mathbb{R}^{n}\right)}\left\|\chi_{B}\right\|_{L^{p^{\prime}(\cdot)\left(\mathbb{R}^{n}\right)}} \leq C .
$$

Lemma 10 (see [29, Lemma 3]). Let $k$ be a positive integer. Then one has that, for all $b \in B M O\left(\mathbb{R}^{n}\right)$ and all $i, j \in \mathbb{Z}$ with $j>i$,

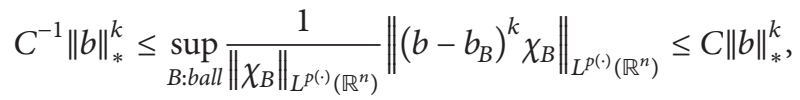

$$
\begin{aligned}
& \left\|\left(b-b_{B_{i}}\right)^{k} \chi_{B_{j}}\right\|_{L^{p(\cdot)}\left(\mathbb{R}^{n}\right)} \leq C(j-i)^{k}\|b\|_{*}^{k}\left\|\chi_{B_{j}}\right\|_{L^{p(\cdot)}\left(\mathbb{R}^{n}\right)} .
\end{aligned}
$$

In fact, if $k=1$, then, from inequalities (23), for all balls $B$ and $i, j \in \mathbb{Z}$ with $j>i$, we have

$$
\begin{gathered}
\left\|\left(b-b_{B}\right) \chi_{B}\right\|_{L^{p(\cdot)}\left(\mathbb{R}^{n}\right)} \leq C\|b\|_{*}\left\|\chi_{B}\right\|_{L^{p(\cdot)}\left(\mathbb{R}^{n}\right)}, \\
\left\|\left(b-b_{B_{i}}\right) \chi_{B_{j}}\right\|_{L^{p(\cdot)}\left(\mathbb{R}^{n}\right)} \leq C(j-i)\|b\|_{*}\left\|\chi_{B_{j}}\right\| \|_{L^{p(\cdot)}\left(\mathbb{R}^{n}\right)} .
\end{gathered}
$$

Lemma 11 (see $\left[1\right.$, Theorem 2.1] ). If $p(\cdot) \in \mathscr{P}\left(\mathbb{R}^{n}\right)$, then, for all $f \in L^{p(\cdot)}\left(\mathbb{R}^{n}\right)$ and all $g \in L^{p^{\prime}(\cdot)}\left(\mathbb{R}^{n}\right)$, one has

$$
\int_{\mathbb{R}^{n}}|f(x) g(x)| d x \leq r_{p}\|f\|_{L^{p(\cdot)}\left(\mathbb{R}^{n}\right)}\|g\|_{L^{p^{\prime}(\cdot)}\left(\mathbb{R}^{n}\right)},
$$

where $r_{p}:=1+1 / p_{+}-1 / p_{-}$. 
4

Journal of Function Spaces

Lemma 12 (see [14, Theorem 2.3]). Let $p, p_{1}, p_{2} \in \mathscr{P}^{0}\left(\mathbb{R}^{n}\right)$ such that $1 / p(x)=1 / p_{1}(x)+1 / p_{2}(x)$. Then there exists a constant $C_{p, p_{1}}$ independent of the functions $f$ and $g$ such that

$$
\|f g\|_{L^{p(\cdot)}\left(\mathbb{R}^{n}\right)} \leq C_{p, p_{1}}\|f\|_{L^{p_{1}(\cdot)}\left(\mathbb{R}^{n}\right)}\|g\|_{L^{p^{(} \cdot()}\left(\mathbb{R}^{n}\right)}
$$

holds for every $f \in L^{p_{1}(\cdot)}\left(\mathbb{R}^{n}\right)$ and $g \in L^{p_{2}(\cdot)}\left(\mathbb{R}^{n}\right)$.

Lemma 13 (see [14, Corollary 2.2]). Let $T$ be a 2-linear Calderón-Zygmund operator and let $b_{1}$ and $b_{2}$ be BMO functions. Let $p(\cdot) \in \mathscr{P}^{0}\left(\mathbb{R}^{n}\right)$ such that there exists $0<p_{*}<$ $p_{-}$with $\left(p(\cdot) / p_{*}\right)^{\prime} \in \mathscr{B}\left(\mathbb{R}^{n}\right)$. If $p_{1}(\cdot), p_{2}(\cdot) \in \mathscr{B}\left(\mathbb{R}^{n}\right)$ such that $1 / p(x)=1 / p_{1}(x)+1 / p_{2}(x)$, then there exists a constant $C$ independent of functions $f_{i} \in L^{p_{i}(\cdot)}\left(\mathbb{R}^{n}\right)$ for $i=1,2$ such that

$$
\left\|\left[b_{1}, b_{2}, T\right]\left(f_{1}, f_{2}\right)\right\|_{L^{p^{(\cdot)}}} \leq C\left\|b_{1}\right\|_{*}\left\|b_{2}\right\|_{*}\left\|f_{1}\right\|_{L^{p_{1}(\cdot)}}\left\|f_{2}\right\|_{L^{p^{2}(\cdot)}} .
$$

Proof of Theorem 6. Although our method is suitable for any multilinear operator, for simplicity, we only consider 2-linear operators. Let $b_{1}$ and $b_{2}$ be BMO functions. Since the set of all bounded compactly supported functions is dense in HernMorrey spaces with variable exponents, we let $f_{1}$ and $f_{2}$ be bounded compactly supported functions; then, for $x \in \mathbb{R}^{n}$, we write

$$
\begin{aligned}
& {\left[b_{1}, b_{2}, T\right]\left(f_{1}, f_{2}\right)(x)} \\
& =b_{1}(x) b_{2}(x) T\left(f_{1}, f_{2}\right)(x)-b_{1}(x) T\left(f_{1}, b_{2} f_{2}\right)(x) \\
& \quad-b_{2}(x) T\left(b_{1} f_{1}, f_{2}\right)(x)+T\left(b_{1} f_{1}, b_{2} f_{2}\right)(x) .
\end{aligned}
$$

We write

$$
f_{i}(x)=\sum_{l_{i}=-\infty}^{\infty} f_{i}(x) \chi_{l_{i}}(x)=: \sum_{l_{i}=-\infty}^{\infty} f_{l_{i}}(x), \quad i=1,2 .
$$

Then, for each $k \in \mathbb{Z}$, if $l_{i} \leq k-1$, from Lemma 11, we obtain

$$
\begin{gathered}
\int_{\mathbb{R}^{n}}\left[b_{i}(x)-b_{i}\left(y_{i}\right)\right] f_{l_{i}}\left(y_{i}\right) d y_{i} \\
\leq C\left[\int_{\mathbb{R}^{n}}\left(b_{i}(x)-b_{B_{l_{i}}}\right) f_{l_{i}}\left(y_{i}\right) d y_{i}\right. \\
\left.\quad+\int_{\mathbb{R}^{n}}\left(b_{B_{l_{i}}}-b_{i}\left(y_{i}\right)\right) f_{l_{i}}\left(y_{i}\right) d y_{i}\right] \\
\leq C\left\|f_{l_{i}}\right\|_{L^{p_{i}(\cdot)}\left(\mathbb{R}^{n}\right)}\left(\left|b_{i}(x)-b_{B_{l_{i}}}\right| \cdot\left\|\chi_{l_{i}}\right\|_{L^{p_{i}^{\prime}(\cdot)}\left(\mathbb{R}^{n}\right)}\right. \\
\left.+\left\|\left|b_{B_{l_{i}}}-b_{i}\right| \cdot \chi_{l_{i}}\right\|_{L^{p^{\prime}(\cdot)}\left(\mathbb{R}^{n}\right)}\right),
\end{gathered}
$$

and by using inequalities (18), (22), and (24), it follows that

$$
\begin{aligned}
& \left\|\int_{\mathbb{R}^{n}}\left[b_{i}(\cdot)-b_{i}\left(y_{i}\right)\right] f_{l_{i}}\left(y_{i}\right) d y_{i} \chi_{k}\right\|_{L^{p_{i}(\cdot)}\left(\mathbb{R}^{n}\right)} \\
& \leq C\left\|f_{l_{i}}\right\|_{L^{p_{i}(\cdot)}\left(\mathbb{R}^{n}\right)}\left(\left\|b_{i}-b_{B_{l_{i}}} \mid \chi_{B_{k}}\right\|_{L^{p_{i}(\cdot)}\left(\mathbb{R}^{n}\right)} \cdot\left\|\chi_{l_{i}}\right\|_{L^{p^{\prime}(\cdot)}\left(\mathbb{R}^{n}\right)}\right. \\
& \left.+\left\|\left|b_{B_{l_{i}}}-b_{i}\right| \chi_{B_{l_{i}}}\right\|_{L^{p_{i}^{\prime}(\cdot)\left(\mathbb{R}^{n}\right)}} \cdot\left\|\chi_{k}\right\|_{L^{p_{i}(\cdot)}\left(\mathbb{R}^{n}\right)}\right) \\
& \leq C\left\|f_{l_{i}}\right\|_{L^{p_{i}(\cdot)}\left(\mathbb{R}^{n}\right)}\left[\left(k-l_{i}\right)\left\|b_{i}\right\|_{*}\left\|\chi_{B_{k}}\right\|_{L^{p_{i}(\cdot)}\left(\mathbb{R}^{n}\right)}\left\|\chi_{l_{i}}\right\|_{L^{p^{\prime}(\cdot)}\left(\mathbb{R}^{n}\right)}\right. \\
& \left.+\left\|b_{i}\right\|_{*}\left\|\chi_{B_{l_{i}}}\right\|_{L^{p_{i}^{\prime} \cdot(\cdot)\left(\mathbb{R}^{n}\right)}}\left\|\chi_{k}\right\|_{L^{p_{i}(\cdot)\left(\mathbb{R}^{n}\right)}}\right] \\
& \leq C\left\|b_{i}\right\|_{*}\left\|f_{l_{i}}\right\|_{L^{p_{i}(\cdot)\left(\mathbb{R}^{n}\right)}}\left(k-l_{i}\right)\left\|\chi_{B_{k}}\right\|_{L^{p_{i}(\cdot)}\left(\mathbb{R}^{n}\right)}\left\|\chi_{B_{l_{i}}}\right\|_{L^{p^{\prime}(\cdot)}\left(\mathbb{R}^{n}\right)} \\
& \leq C\left\|b_{i}\right\|_{*}\left\|f_{l_{i}}\right\|_{L^{p_{i}(\cdot)}\left(\mathbb{R}^{n}\right)}\left(k-l_{i}\right)\left\|\chi_{B_{k}}\right\|_{L^{p_{i}(\cdot)}\left(\mathbb{R}^{n}\right)} \\
& \times\left\|\chi_{B_{k}}\right\|_{L^{p_{i}^{\prime}(\cdot)}\left(\mathbb{R}^{n}\right)} \frac{\left\|\chi_{B_{l_{i}}}\right\|_{L^{p_{i}^{\prime} \cdot(\cdot)}\left(\mathbb{R}^{n}\right)}}{\left\|\chi_{B_{k}}\right\|_{L^{p_{i}^{\prime} \cdot(\cdot)}\left(\mathbb{R}^{n}\right)}} \\
& \leq C\left(k-l_{i}\right) 2^{\left(l_{i}-k\right) n \delta_{2}} 2^{k n}\left\|b_{i}\right\|_{*}\left\|f_{l_{i}}\right\|_{L^{p_{i}(\cdot)}\left(\mathbb{R}^{n}\right)^{n}} .
\end{aligned}
$$

Similarly, if $l_{i} \geq k+1$, we have

$$
\begin{aligned}
& \left\|\int_{\mathbb{R}^{n}}\left[b_{i}(\cdot)-b_{i}\left(y_{i}\right)\right] f_{l_{i}}\left(y_{i}\right) d y_{i} \chi_{k}\right\|_{L^{p_{i}(\cdot)}\left(\mathbb{R}^{n}\right)} \\
& \leq C\left\|f_{l_{i}}\right\|_{L^{p_{i}(\cdot)\left(\mathbb{R}^{n}\right)}}\left(\left\|\left|b_{i}-b_{B_{k}}\right| \chi_{B_{k}}\right\|_{L^{p^{i}(\cdot)}\left(\mathbb{R}^{n}\right)} \cdot\left\|\chi_{l_{i}}\right\|_{L^{p_{i}^{\prime} \cdot(\cdot)}\left(\mathbb{R}^{n}\right)}\right. \\
& \left.+\left\|\left|b_{B_{k}}-b_{i}\right| \chi_{B_{l_{i}}}\right\|_{L^{p_{i}^{\prime}(\cdot)}\left(\mathbb{R}^{n}\right)} \cdot\left\|\chi_{k}\right\|_{L^{p_{i}(\cdot)}\left(\mathbb{R}^{n}\right)}\right) \\
& \leq C\left\|f_{l_{i}}\right\|_{L^{p(\cdot)}\left(\mathbb{R}^{n}\right)}\left[\left\|b_{i}\right\|_{*}\left\|\chi_{B_{k}}\right\|_{L^{p_{i}(\cdot)}\left(\mathbb{R}^{n}\right)}\left\|\chi_{l_{i}}\right\|_{L^{p_{i}^{\prime}(\cdot)}\left(\mathbb{R}^{n}\right)}\right. \\
& \left.+\left(l_{i}-k\right)\left\|b_{i}\right\|_{*}\left\|\chi_{B_{l_{i}}}\right\|_{L^{p_{i}^{\prime} \cdot(\cdot)}\left(\mathbb{R}^{n}\right)}\left\|\chi_{k}\right\|_{L^{p_{i}(\cdot)}\left(\mathbb{R}^{n}\right)}\right] \\
& \leq C\left\|b_{i}\right\|_{*}\left\|f_{l_{i}}\right\|_{L^{p_{i}(\cdot)}\left(\mathbb{R}^{n}\right)}\left(l_{i}-k\right)\left\|\chi_{B_{k}}\right\|_{L^{p_{i}(\cdot)}\left(\mathbb{R}^{n}\right)}\left\|\chi_{B_{l_{i}}}\right\|_{L^{p_{i}^{\prime}(\cdot)}\left(\mathbb{R}^{n}\right)} \\
& \leq C\left\|b_{i}\right\|_{*}\left\|f_{l_{i}}\right\|_{L^{p_{i}(\cdot)}\left(\mathbb{R}^{n}\right)}\left(l_{i}-k\right)
\end{aligned}
$$

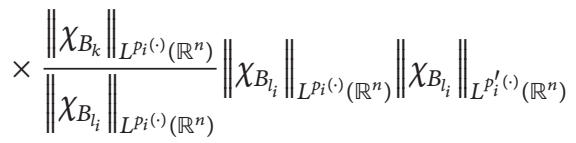

$$
\begin{aligned}
& \leq C\left(l_{i}-k\right) 2^{\left(k-l_{i}\right) n \delta_{1}} 2^{l_{i} n}\left\|b_{i}\right\|_{*}\left\|f_{l_{i}}\right\|_{L^{p_{i}(\cdot)}\left(\mathbb{R}^{n}\right)} .
\end{aligned}
$$

As for the case $l_{i}=k$, we get

$$
\begin{aligned}
\left\|\int_{\mathbb{R}^{n}}\left[b_{i}(\cdot)-b_{i}\left(y_{i}\right)\right] f_{l_{i}}\left(y_{i}\right) d y_{i} \chi_{k}\right\|_{L^{p_{i}} \cdot(\cdot)\left(\mathbb{R}^{n}\right)} \\
\leq C\left\|f_{l_{i}}\right\|_{L^{p_{i}(\cdot)\left(\mathbb{R}^{n}\right)}}\left(\left\|\left|b_{i}-b_{B_{k}}\right| \chi_{B_{k}}\right\|_{L^{p_{i}(\cdot)}\left(\mathbb{R}^{n}\right)} \cdot\left\|\chi_{l_{i}}\right\|_{L^{p^{\prime}(\cdot)}\left(\mathbb{R}^{n}\right)}\right. \\
\left.+\left\|\left|b_{B_{k}}-b_{i}\right| \chi_{B_{l_{i}}}\right\|_{L^{p_{i}^{\prime} \cdot(\cdot)}\left(\mathbb{R}^{n}\right)} \cdot\left\|\chi_{k}\right\|_{L_{L^{p_{i}}(\cdot)\left(\mathbb{R}^{n}\right)}}\right)
\end{aligned}
$$




$$
\begin{aligned}
& \leq C\left\|b_{i}\right\|_{*}\left\|f_{l_{i}}\right\|_{L^{p_{i} \cdot(\cdot)}\left(\mathbb{R}^{n}\right)}\left\|\chi_{B_{k}}\right\|_{L^{p_{i} \cdot(\cdot)}\left(\mathbb{R}^{n}\right)}\left\|\chi_{B_{k}}\right\|_{L^{p_{i}^{\prime}(\cdot)}\left(\mathbb{R}^{n}\right)} \\
& \leq C 2^{k n}\left\|b_{i}\right\|_{*}\left\|f_{l_{i}}\right\|_{L^{p_{i} \cdot(\cdot)}\left(\mathbb{R}^{n}\right)^{n}} \cdot
\end{aligned}
$$

By Lemma 8, we know

$$
\left\|\left[b_{1}, b_{2}, T\right]\left(f_{1}, f_{2}\right)\right\|_{M \dot{K}_{q, P(\cdot)}^{\alpha(\cdot), \lambda}\left(\mathbb{R}^{n}\right)} \approx \max \{E, F\},
$$

where

$$
\begin{aligned}
& E:=\sup _{L \leq 0, L \in \mathbb{Z}} 2^{-L \lambda} \\
& \times\left(\sum_{k=-\infty}^{L} 2^{k \alpha(0) q} \| \sum_{l_{1}=-\infty}^{\infty} \sum_{l_{2}=-\infty}^{\infty}\left[b_{1}, b_{2}, T\right]\right. \\
& \left.\times\left(f_{l_{1}}, f_{l_{2}}\right) \chi_{k} \|_{L^{p(\cdot)}\left(\mathbb{R}^{n}\right)}^{q}\right)^{1 / q}, \\
& :=\sup _{L>0, L \in \mathbb{Z}}\left[2^{-L \lambda}\left(\sum_{k=-\infty}^{-1} 2^{k \alpha(0) q}\left\|\left[b_{1}, b_{2}, T\right]\left(f_{1}, f_{2}\right) \chi_{k}\right\|_{L^{p(\cdot)}\left(\mathbb{R}^{n}\right)}^{q}\right)^{1 / q}\right. \\
& +2^{-L \lambda}\left(\sum_{k=0}^{L} 2^{k \alpha_{\infty} q}\right. \\
& \left.\left.\times\left\|\left[b_{1}, b_{2}, T\right]\left(f_{1}, f_{2}\right) \chi_{k}\right\|_{L^{p \cdot\left(\cdot\left(\mathbb{R}^{n}\right)\right.}}^{q}\right)^{1 / q}\right] .
\end{aligned}
$$

Since the estimate of $F$ is essentially similar to that of $E$, so it suffices to prove that $E$ is bounded in Herz-Morrey spaces with variable exponents. It is easy to see that

$$
E \leq C \sum_{i=1}^{9} I_{i}
$$

where

$$
\begin{aligned}
I_{1}:=\sup _{L \leq 0, L \in \mathbb{Z}} 2^{-L \lambda} & \\
& \times\left(\sum_{k=-\infty}^{L} 2^{k \alpha(0) q} \| \sum_{l_{1}=-\infty}^{k-2} \sum_{l_{2}=-\infty}^{k-2}\left[b_{1}, b_{2}, T\right]\right. \\
& \left.\quad \times\left(f_{l_{1}}, f_{l_{2}}\right) \chi_{k} \|_{L^{p(\cdot)}\left(\mathbb{R}^{n}\right)}^{q}\right)^{1 / q},
\end{aligned}
$$

$$
\begin{aligned}
I_{2}:= & \sup _{L \leq 0, L \in \mathbb{Z}} 2^{-L \lambda} \\
& \times\left(\sum_{k=-\infty}^{L} 2^{k \alpha(0) q} \| \sum_{l_{1}=-\infty}^{k-2} \sum_{l_{2}=k-1}^{k+1}\left[b_{1}, b_{2}, T\right]\right.
\end{aligned}
$$

$$
\left.\times\left(f_{l_{1}}, f_{l_{2}}\right) \chi_{k} \|_{L^{p(\cdot)}\left(\mathbb{R}^{n}\right)}^{q}\right)^{1 / q},
$$

$$
I_{3}:=\sup _{L \leq 0, L \in \mathbb{Z}} 2^{-L \lambda}
$$

$$
\times\left(\sum_{k=-\infty}^{L} 2^{k \alpha(0) q} \| \sum_{l_{1}=-\infty}^{k-2} \sum_{l_{2}=k+2}^{\infty}\left[b_{1}, b_{2}, T\right]\right.
$$

$$
\left.\times\left(f_{l_{1}}, f_{l_{2}}\right) \chi_{k} \|_{L^{p(\cdot)}\left(\mathbb{R}^{n}\right)}^{q}\right)^{1 / q},
$$

$$
\begin{aligned}
I_{4}:= & \sup _{L \leq 0, L \in \mathbb{Z}} 2^{-L \lambda} \\
& \times\left(\sum_{k=-\infty}^{L} 2^{k \alpha(0) q} \|_{l_{1}=k-1}^{k+1} \sum_{l_{2}=-\infty}^{k-2}\left[b_{1}, b_{2}, T\right]\right.
\end{aligned}
$$

$$
\left.\times\left(f_{l_{1}}, f_{l_{2}}\right) \chi_{k} \|_{L^{p(\cdot)}\left(\mathbb{R}^{n}\right)}^{q}\right)^{1 / q},
$$

$$
\begin{aligned}
I_{5}:= & \sup _{L \leq 0, L \in \mathbb{Z}} 2^{-L \lambda} \\
& \times\left(\sum_{k=-\infty}^{L} 2^{k \alpha(0) q} \| \sum_{l_{1}=k-1}^{k+1} \sum_{l_{2}=k-1}^{k+1}\left[b_{1}, b_{2}, T\right]\right.
\end{aligned}
$$

$$
\left.\times\left(f_{l_{1}}, f_{l_{2}}\right) \chi_{k} \|_{L^{p(\cdot)}\left(\mathbb{R}^{n}\right)}^{q}\right)^{1 / q},
$$

$$
\begin{aligned}
& I_{6}:= \sup _{L \leq 0, L \in \mathbb{Z}} 2^{-L \lambda} \\
& \times\left(\sum_{k=-\infty}^{L} 2^{k \alpha(0) q} \| \sum_{l_{1}=k-1}^{k+1} \sum_{l_{2}=k+2}^{\infty}\left[b_{1}, b_{2}, T\right]\right. \\
&\left.\times\left(f_{l_{1}}, f_{l_{2}}\right) \chi_{k} \|_{L^{p(\cdot)}\left(\mathbb{R}^{n}\right)}^{q}\right)^{1 / q}, \\
& I_{7}:= \sup _{L \leq 0, L \in \mathbb{Z}} 2^{-L \lambda}, \\
& \times\left(\sum_{k=-\infty}^{L} 2^{k \alpha(0) q} \| \sum_{l_{1}=k+2}^{\infty} \sum_{l_{2}=-\infty}^{k-2}\left[b_{1}, b_{2}, T\right]\right. \\
&\left.\times\left(f_{l_{1}}, f_{l_{2}}\right) \chi_{k} \|_{L^{p^{(\cdot)}\left(\mathbb{R}^{n}\right)}}^{q}\right)^{1 / q},
\end{aligned}
$$




$$
\begin{aligned}
& I_{8}:= \sup _{L \leq 0, L \in \mathbb{Z}} 2^{-L \lambda} \\
& \times\left(\sum_{k=-\infty}^{L} 2^{k \alpha(0) q} \| \sum_{l_{1}=k+2}^{\infty} \sum_{l_{2}=k-1}^{k+1}\left[b_{1}, b_{2}, T\right]\right. \\
& I_{9}:= \sup _{L \leq 0, L \in \mathbb{Z}} 2^{-L \lambda} \\
& \times\left(\sum_{k=-\infty}^{L} 2^{k \alpha(0) q}\left\|\sum_{l_{1}=k+2}^{\infty} \sum_{l_{2}=k+2}^{\infty}\left[b_{l_{2}}\right) \chi_{k}\right\|_{L^{p(\cdot)}\left(\mathbb{R}^{n}\right)}\right)^{1 / q}, T \\
&\left.\times\left(f_{l_{1}}, f_{l_{2}}\right) \chi_{k} \|_{L^{p(\cdot)}\left(\mathbb{R}^{n}\right)}^{q}\right)^{1 / q} .
\end{aligned}
$$

Using the symmetry of $f_{1}$ and $f_{2}$, we only need to estimate $I_{1}, I_{2}, I_{3}, I_{5}, I_{6}, I_{9}$ because the estimates of $I_{2}, I_{3}$, and $I_{6}$ are analogous to those of $I_{4}, I_{7}$, and $I_{8}$, respectively. In what follows, we divide it into 6 steps.

Step 1. To estimate the term of $I_{1}$, we note that $l_{i} \leq k-2$ for $i=1,2$. Thus, for $x \in D_{k}, y_{i} \in D_{l_{i}}$,

$$
\left|x-y_{i}\right| \geq|| x|-| y_{i}||>2^{k-1}-2^{l_{i}} \geq 2^{k-2} \text {. }
$$

Then, for $x \in D_{k}$, we get

$$
\left|K\left(x, y_{1}, y_{2}\right)\right| \leq C\left(\left|x-y_{1}\right|+\left|x-y_{2}\right|\right)^{-2 n} \leq C 2^{-2 k n} \text {. }
$$

Therefore,

$$
\begin{aligned}
& {\left[b_{1}, b_{2}, T\right]\left(f_{l_{1}}, f_{l_{2}}\right)(x)} \\
& \quad \leq C 2^{-2 k n} \prod_{i=1}^{2} \int_{\mathbb{R}^{n}}\left[b_{i}(x)-b_{i}\left(y_{i}\right)\right] f_{l_{i}}\left(y_{i}\right) d y_{i} .
\end{aligned}
$$

By Lemma 12 and inequality (31), we obtain

$$
\begin{aligned}
& \left\|\sum_{l_{1}=-\infty}^{k-2} \sum_{l_{2}=-\infty}^{k-2}\left[b_{1}, b_{2}, T\right]\left(f_{l_{1}}, f_{l_{2}}\right) \chi_{k}\right\|_{L^{p(\cdot)}\left(\mathbb{R}^{n}\right)} \\
& \leq C\left\|2^{-2 k n} \prod_{i=1}^{2}\left(\sum_{l_{i}=-\infty}^{k-2} \int_{\mathbb{R}^{n}}\left[b_{i}(\cdot)-b_{i}\left(y_{i}\right)\right] f_{l_{i}}\left(y_{i}\right) d y_{i}\right) \chi_{k}\right\|_{L^{p(\cdot)}\left(\mathbb{R}^{n}\right)} \\
& \leq C 2^{-2 k n} \prod_{i=1}^{2} \sum_{l_{i}=-\infty}^{k-2}\left\|\int_{\mathbb{R}^{n}}\left[b_{i}(\cdot)-b_{i}\left(y_{i}\right)\right] f_{l_{i}}\left(y_{i}\right) d y_{i} \chi_{k}\right\|_{L^{p_{i}(\cdot)}\left(\mathbb{R}^{n}\right)} \\
& \leq C \prod_{i=1}^{2} \sum_{l_{i}=-\infty}^{k-2}\left(k-l_{i}\right) 2^{\left(l_{i}-k\right) n \delta_{2}}\left\|b_{i}\right\|_{*}\left\|f_{l_{i}}\right\|_{L^{p_{i}(\cdot)}\left(\mathbb{R}^{n}\right)}
\end{aligned}
$$

Since $1 / q=1 / q_{1}+1 / q_{2}$, it follows that

$$
\begin{aligned}
& I_{1} \leq C \sup _{L \leq 0, L \in \mathbb{Z}} 2^{-L \lambda} \\
& \times\left\{\sum _ { k = - \infty } ^ { L } 2 ^ { k \alpha ( 0 ) q } \left(\prod_{i=1}^{2} \sum_{l_{i}=-\infty}^{k-2}\left(k-l_{i}\right) 2^{\left(l_{i}-k\right) n \delta_{2}}\left\|b_{i}\right\|_{*}\right.\right. \\
&\left.\left.\times\left\|f_{l_{i}}\right\|_{L^{p_{i}(\cdot)}\left(\mathbb{R}^{n}\right)}\right)\right\}^{1 / q} \\
& \leq C \sup _{L \leq 0, L \in \mathbb{Z}} \prod_{i=1}^{2} 2^{-L \lambda_{i}}\left\|b_{i}\right\|_{*} \\
& \times\left\{\sum _ { k = - \infty } ^ { L } \left(\sum_{l_{i}=-\infty}^{k-2}\left(k-l_{i}\right) 2^{\left(l_{i}-k\right) n \delta_{2}+k \alpha_{i}(0)}\right.\right. \\
&= C \sup _{L \leq 0, L \in \mathbb{Z}} I_{11}(L) I_{12}(L), \\
&\left.\left.\times f_{l_{i}} \|_{L^{p_{i}(\cdot)}\left(\mathbb{R}^{n}\right)}\right)^{q_{i}}\right\}^{1 / q_{i}}
\end{aligned}
$$

where

$$
\begin{aligned}
I_{1 i}(L):= & 2^{-L \lambda_{i}}\left\|b_{i}\right\|_{*} \\
& \times\left\{\sum _ { k = - \infty } ^ { L } \left(\sum_{l_{i}=-\infty}^{k-2}\left(k-l_{i}\right) 2^{\left(l_{i}-k\right) \mu_{i}} 2^{l_{i} \alpha_{i}(0)}\right.\right. \\
& \left.\left.\times\left\|f_{l_{i}}\right\|_{L^{p_{i}(\cdot)}\left(\mathbb{R}^{n}\right)}\right)^{q_{i}}\right\}^{1 / q_{i}}
\end{aligned}
$$

Here, we denote $\mu_{i}=n \delta_{2}-\alpha_{i}(0)$ for short. It follows from condition (19) that $\mu_{i}>0$. To continue calculations for (43), we consider the two cases $0<q_{i} \leq 1$ and $1<q_{i}<\infty$. If $0<q_{i} \leq 1$, since, for $a_{1}, a_{2}, \ldots \geq 0$,

$$
\left(\sum_{h=1}^{\infty} a_{h}\right)^{q_{i}} \leq \sum_{h=1}^{\infty} a_{h}^{q_{i}},
$$

then

$$
\begin{gathered}
I_{1 i}(L) \\
\leq C 2^{-L \lambda_{i}}\left\|b_{i}\right\|_{*}\left\{\sum_{k=-\infty}^{L} \sum_{l_{i}=-\infty}^{k-2}\left(k-l_{i}\right)^{q_{i}} 2^{\left(l_{i}-k\right) \mu_{i} q_{i}} 2^{l_{i} \alpha_{i}(0)^{q_{i}}}\right. \\
\left.\times\left\|f_{l_{i}}\right\|_{L^{p_{i}(\cdot)}\left(\mathbb{R}^{n}\right)}^{q_{i}}\right\}^{1 / q_{i}}
\end{gathered}
$$




$$
\begin{aligned}
& =C 2^{-L \lambda_{i}}\left\|b_{i}\right\|_{*}\left\{\sum_{l_{i}=-\infty}^{L-2} 2^{l_{i} \alpha_{i}(0)^{q_{i}}}\left\|f_{l_{i}}\right\|_{L^{p_{i}(\cdot)}\left(\mathbb{R}^{n}\right)}^{q_{i}}\right. \\
& \left.\times \sum_{k=l_{i}+2}^{L}\left(k-l_{i}\right)^{q_{i}} 2^{\left(l_{i}-k\right) \mu_{i} q_{i}}\right\}^{1 / q_{i}} \\
& \leq C\left\|b_{i}\right\|_{*} 2^{-L \lambda_{i}}\left\{\sum_{l_{i}=-\infty}^{L-2} 2^{l_{i} \alpha_{i}(0){ }^{q_{i}}}\left\|f_{l_{i}}\right\|_{L^{p_{i}(\cdot)}\left(\mathbb{R}^{n}\right)}^{q_{i}}\right\}^{1 / q_{i}} \\
& \leq C\left\|b_{i}\right\|_{*}\left\|f_{l_{i}}\right\|_{M \dot{K}_{q_{i} \alpha_{i}(\cdot),(\cdot)}^{\alpha_{i}}\left(\mathbb{R}^{n}\right)^{n}} .
\end{aligned}
$$

If $1<q_{i}<\infty$, by the Hölder inequality, we obtain

$$
\begin{aligned}
& I_{1 i}(L)=2^{-L \lambda_{i}}\left\|b_{i}\right\|_{*} \\
& \times\left\{\sum _ { k = - \infty } ^ { L } \left(\sum_{l_{i}=-\infty}^{k-2}\left(k-l_{i}\right) 2^{\left(l_{i}-k\right) \mu_{i}} 2^{l_{i} \alpha_{i}(0)}\right.\right. \\
& \left.\left.\times\left\|f_{l_{i}}\right\|_{L^{p_{i} \cdot(\cdot)}\left(\mathbb{R}^{n}\right)}\right)^{q_{i}}\right\}^{1 / q_{i}} \\
& \leq C 2^{-L \lambda_{i}}\left\|b_{i}\right\|_{*} \\
& \times\left\{\sum_{k=-\infty}^{L}\left(\sum_{l_{i}=-\infty}^{k-2} 2^{\left(l_{i}-k\right) \mu_{i}\left(q_{i} / 2\right)} 2^{l_{i} \alpha_{i}(0) q_{i}}\left\|f_{l_{i}}\right\|_{L^{p_{i}} \cdot(\cdot)\left(\mathbb{R}^{n}\right)}^{q_{i}}\right)\right. \\
& \left.\times\left(\sum_{l_{i}=-\infty}^{k-2}\left(k-l_{i}\right)^{q_{i}^{\prime}} 2^{\left(l_{i}-k\right) \mu_{i}\left(q_{i}^{\prime} / 2\right)}\right)^{q_{i} / q_{i}^{\prime}}\right\}^{1 / q_{i}} \\
& \leq C 2^{-L \lambda_{i}}\left\|b_{i}\right\|_{*} \\
& \times\left\{\sum_{k=-\infty}^{L}\left(\sum_{l_{i}=-\infty}^{k-2} 2^{\left(l_{i}-k\right) \mu_{i}\left(q_{i} / 2\right)} 2^{l_{i} \alpha_{i}(0) q_{i}}\left\|f_{l_{i}}\right\|_{L^{p_{i}(\cdot)}\left(\mathbb{R}^{n}\right)}^{q_{i}}\right)\right\}^{1 / q_{i}} \\
& =C 2^{-L \lambda_{i}}\left\|b_{i}\right\|_{*}\left(\sum_{l_{i}=-\infty}^{L-2} 2^{l_{i} \alpha_{i}(0) q_{i}}\right. \\
& \left.\times\left\|f_{l_{i}}\right\|_{L^{p_{i}(\cdot)\left(\mathbb{R}^{n}\right)}}^{q_{i}} \sum_{k=l_{i}+2}^{L} 2^{\left(l_{i}-k\right) \mu_{i}\left(q_{i} / 2\right)}\right)^{1 / q_{i}} \\
& \leq C\left\|b_{i}\right\|_{*} 2^{-L \lambda_{i}}\left(\sum_{l_{i}=-\infty}^{L-2} 2^{l_{i} \alpha_{i}(0) q_{i}}\left\|f_{l_{i}}\right\|_{L^{p_{i}(\cdot)}\left(\mathbb{R}^{n}\right)}^{q_{q}}\right)^{1 / q_{i}} \\
& \leq C\left\|b_{i}\right\|_{*}\left\|f_{i}\right\|_{M \dot{K}_{q_{i}, p_{i} \cdot(\cdot)}^{\alpha_{i}(\cdot) \lambda_{i}}\left(\mathbb{R}^{n}\right)} .
\end{aligned}
$$

Thus, for any $0<q_{i}<\infty$,

$$
\begin{aligned}
I_{1} & \leq C \sup _{L \leq 0, L \in \mathbb{Z}} I_{11}(L) I_{12}(L) \\
& \leq C\left\|b_{1}\right\|_{*}\left\|b_{2}\right\|_{*}\left\|f_{1}\right\|_{M \dot{K}_{q_{1}, p_{1}(\cdot)}^{\alpha_{1}\left(\cdot, \lambda_{1}\right.}\left(\mathbb{R}^{n}\right)}\left\|f_{2}\right\|_{M \dot{K}_{q_{2}, p_{2}(\cdot)}^{\alpha_{2}(\cdot), \lambda_{2}}\left(\mathbb{R}^{n}\right)} .
\end{aligned}
$$

Step 2. To estimate $I_{2}$, for $x \in D_{k}, y_{i} \in D_{l_{i}}, i=1,2$ and $l_{1} \leq k-2, k-1 \leq l_{2} \leq k+1$, we have

$$
\left|x-y_{2}\right| \geq\left|x-y_{1}\right| \geq|| x|-| y_{1}||>2^{k-2} .
$$

Since $l_{1} \leq k-2$, it follows from inequality (31) that

$$
\begin{aligned}
& \left\|\sum_{l_{1}=-\infty}^{k-2} \sum_{l_{2}=k-1}^{k+1}\left[b_{1}, b_{2}, T\right]\left(f_{l_{1}}, f_{l_{2}}\right) \chi_{k}\right\|_{L^{p^{(\cdot)}\left(\mathbb{R}^{n}\right)}} \\
& \leq C 2^{-2 k n} \sum_{l_{1}=-\infty}^{k-2}\left\|\int_{\mathbb{R}^{n}}\left[b_{1}(\cdot)-b_{1}\left(y_{1}\right)\right] f_{l_{1}}\left(y_{1}\right) d y_{1}\right\|_{L^{p_{1}(\cdot)}\left(\mathbb{R}^{n}\right)} \\
& \quad \times \sum_{l_{2}=k-1}^{k+1}\left\|\int_{\mathbb{R}^{n}}\left[b_{2}(\cdot)-b_{2}\left(y_{2}\right)\right] f_{l_{2}}\left(y_{2}\right) d y_{2} \chi_{k}\right\|_{L^{p_{2}(\cdot)}\left(\mathbb{R}^{n}\right)} \\
& \leq C\left(\sum_{l_{1}=-\infty}^{k-2}\left(k-l_{1}\right) 2^{\left(l_{1}-k\right) n \delta_{2}}\left\|b_{1}\right\|_{*}\left\|f_{l_{1}}\right\|_{L^{p_{1}(\cdot)}\left(\mathbb{R}^{n}\right)}\right) \\
& \quad \times 2^{-k n} \sum_{l_{2}=k-1}^{k+1}\left\|\int_{\mathbb{R}^{n}}\left[b_{2}(\cdot)-b_{2}\left(y_{2}\right)\right] f_{l_{2}}\left(y_{2}\right) d y_{2} \chi_{k}\right\|_{L^{p_{2}(\cdot)}\left(\mathbb{R}^{n}\right)} .
\end{aligned}
$$

For $k-1 \leq l_{2} \leq k+1$, combining the above term and inequalities (32) and (33), we have

$$
\begin{aligned}
& I_{2} \leq C \sup _{L \leq 0, L \in \mathbb{Z}} 2^{-L \lambda}\left\|b_{1}\right\|_{*}\left\|b_{2}\right\|_{*} \\
& \quad \times\left\{\sum _ { k = - \infty } ^ { L } 2 ^ { k \alpha ( 0 ) q } \left(\sum_{l_{1}=-\infty}^{k-2} \sum_{l_{2}=k-1}^{k+1}\left(k-l_{1}\right) 2^{\left(l_{1}-k\right) n \delta_{2}}\right.\right. \\
& \left.\left.\times\left\|f_{l_{1}}\right\|_{L^{p_{1}(\cdot)\left(\mathbb{R}^{n}\right)}} 2^{\left(l_{2}-k\right) n}\left\|f_{l_{2}}\right\|_{L^{p_{2} \cdot(\cdot)}\left(\mathbb{R}^{n}\right)}\right)^{q}\right\}^{1 / q} \\
& \leq C \sup _{L \leq 0, L \in \mathbb{Z}} 2^{-L \lambda_{1}}\left\|b_{1}\right\|_{*} \\
& \times\left\{\sum _ { k = - \infty } ^ { L } \left(\sum_{l_{1}=-\infty}^{k-2}\left(k-l_{1}\right) 2^{\left(l_{1}-k\right) \mu_{1}} 2^{l_{1} \alpha_{1}(0)}\right.\right. \\
& \left.\left.\times\left\|f_{l_{1}}\right\|_{L^{p_{1} \cdot(\cdot)}\left(\mathbb{R}^{n}\right)}\right)^{q_{1}}\right\}^{1 / q_{1}}
\end{aligned}
$$




$$
\begin{aligned}
& \quad \times 2^{-L \lambda_{2}}\left\|b_{2}\right\|_{*}\left\{\sum _ { k = - \infty } ^ { L } \left(\sum_{l_{2}=k-1}^{k+1} 2^{k \alpha_{2}(0)} 2^{\left(l_{2}-k\right) n}\right.\right. \\
& \left.\left.\quad \times\left\|f_{l_{2}}\right\|_{L^{p_{2}(\cdot)}\left(\mathbb{R}^{n}\right)}\right)^{q_{2}}\right\}^{1 / q_{2}} \\
& =: C \sup _{L \leq 0, L \in \mathbb{Z}} I_{21}(L) I_{22}(L) .
\end{aligned}
$$

Here, we used $2^{-n \delta_{2}}<1$ and $2^{\left(l_{2}-k\right) n\left(1-\delta_{1}\right)}<2^{\left(l_{2}-k\right) n}$ in the first inequality.

Obviously,

$$
I_{21}(L)=I_{11}(L) \leq C\left\|b_{1}\right\|_{*}\left\|f_{1}\right\|_{M \dot{K}_{q_{1}, p_{1} \cdot(\cdot)}^{\alpha_{1}(\cdot) \lambda_{1}}\left(\mathbb{R}^{n}\right)}
$$

Therefore, we only need to estimate $I_{22}(L)$ :

$$
\begin{aligned}
& I_{22}(L) \\
& =2^{-L \lambda_{2}}\left\|b_{2}\right\|_{*}\left\{\sum_{k=-\infty}^{L} 2^{k \alpha_{2}(0)^{q_{2}}}\right. \\
& \left.\times\left(\sum_{l_{2}=k-1}^{k+1} 2^{\left(l_{2}-k\right) n}\left\|f_{l_{2}}\right\|_{L^{p_{2}(\cdot)\left(\mathbb{R}^{n}\right)}}\right)^{q_{2}}\right\}^{1 / q_{2}} \\
& \leq C\left\|b_{2}\right\|_{*} 2^{-L \lambda_{2}}\left(\sum_{k=-\infty}^{L} 2^{k \alpha_{2}(0)^{q_{2}}}\left\|f_{2} \chi_{k}\right\|_{L^{p_{2}(\cdot)}\left(\mathbb{R}^{n}\right)}^{q_{q_{2}}}\right)^{1 / q_{2}} \\
& \leq C\left\|b_{2}\right\|_{*}\left\|f_{2}\right\|_{M \dot{K}_{q_{2}, p_{2}(\cdot)}^{\alpha_{2}(\cdot), \lambda_{2}}\left(\mathbb{R}^{n}\right)} \text {. }
\end{aligned}
$$

Step 3. To estimate $I_{3}$, for $x \in D_{k}, y_{i} \in D_{l_{i}}, i=1,2$, and $l_{1} \leq k-2, l_{2} \geq k+2$, then we have

$$
\left|x-y_{1}\right| \geq|x|-\left|y_{1}\right|>2^{k-2}, \quad\left|x-y_{2}\right| \geq\left|y_{2}\right|-|x|>2^{l_{2}-2} .
$$

Thus, for $x \in C_{k}$, we get

$$
\begin{aligned}
& {\left[b_{1}, b_{2}, T\right]\left(f_{l_{1}}, f_{l_{2}}\right)(x)} \\
& \leq C 2^{-k n} \int_{\mathbb{R}^{n}}\left[b_{1}(x)-b_{1}\left(y_{1}\right)\right] f_{l_{1}}\left(y_{1}\right) d y_{1} 2^{-l_{2} n} \\
& \quad \times \int_{\mathbb{R}^{n}}\left[b_{2}(x)-b_{2}\left(y_{2}\right)\right] f_{l_{2}}\left(y_{2}\right) d y_{2} .
\end{aligned}
$$

From inequalities (31) and (32), we obtain

$$
\begin{aligned}
& \left\|\sum_{l_{1}=-\infty}^{k-2} \sum_{l_{2}=k+2}^{\infty}\left[b_{1}, b_{2}, T\right]\left(f_{l_{1}}, f_{l_{2}}\right) \chi_{k}\right\|_{L^{p(\cdot)}\left(\mathbb{R}^{n}\right)} \\
& \leq C \sum_{l_{1}=-\infty}^{k-2}\left(k-l_{1}\right) 2^{\left(l_{1}-k\right) n \delta_{2}}\left\|b_{1}\right\|_{*}\left\|f_{l_{1}}\right\|_{L^{p_{1}(\cdot)}\left(\mathbb{R}^{n}\right)} \\
& \quad \times \sum_{l_{2}=k+2}^{\infty}\left(l_{2}-k\right) 2^{\left(k-l_{2}\right) n \delta_{1}}\left\|b_{2}\right\|_{*}\left\|f_{l_{2}}\right\|_{L^{p_{2}(\cdot)}\left(\mathbb{R}^{n}\right)^{n}} .
\end{aligned}
$$

Consequently, it follows that

$$
\begin{aligned}
& I_{3} \leq C \sup _{L \leq 0, L \in \mathbb{Z}} 2^{-L \lambda}\left\|b_{1}\right\|_{*}\left\|b_{2}\right\|_{*} \\
& \times\left\{\sum_{k=-\infty}^{L} 2^{k \alpha(0) q}\left(\sum_{l_{1}=-\infty}^{k-2}\left(k-l_{1}\right) 2^{\left(l_{1}-k\right) n \delta_{2}}\left\|f_{l_{1}}\right\|_{L^{p_{1}(\cdot)}\left(\mathbb{R}^{n}\right)}\right)^{q}\right. \\
& \left.\times\left(\sum_{l_{2}=k+2}^{\infty}\left(l_{2}-k\right) 2^{\left(k-l_{2}\right) n \delta_{1}}\left\|f_{l_{2}}\right\|_{L^{p_{2}(\cdot)}\left(\mathbb{R}^{n}\right)}\right)^{q}\right\}^{1 / q} \\
& \leq C \sup _{L \leq 0, L \in \mathbb{Z}} 2^{-L \lambda_{1}}\left\|b_{1}\right\|_{*} \\
& \times\left\{\sum _ { k = - \infty } ^ { L } \left(\sum_{l_{1}=-\infty}^{k-2}\left(k-l_{1}\right) 2^{\left(l_{1}-k\right) \mu_{1}} 2^{l_{1} \alpha_{1}(0)}\right.\right. \\
& \left.\left.\times\left\|f_{l_{1}}\right\|_{L^{p_{1}(\cdot)}\left(\mathbb{R}^{n}\right)}\right)^{q_{1}}\right\}^{1 / q_{1}} \\
& \times 2^{-L \lambda_{2}}\left\|b_{2}\right\|_{*} \\
& \times\left\{\sum _ { k = - \infty } ^ { L } \left(\sum_{l_{2}=k+2}^{\infty}\left(l_{2}-k\right)\right.\right. \\
& \left.\left.\times 2^{\left(k-l_{2}\right)\left(n \delta_{1}+\alpha_{2}(0)\right)} 2^{l_{2} \alpha_{2}(0)}\left\|f_{l_{2}}\right\|_{L^{p_{2}(\cdot)}\left(\mathbb{R}^{n}\right)}\right)^{q_{2}}\right\}^{1 / q_{2}} \\
& =: C \sup _{L \leq 0, L \in \mathbb{Z}} I_{31}(L) I_{32}(L) .
\end{aligned}
$$

Note that

$$
I_{31}(L)=I_{21}(L) \leq C\left\|b_{1}\right\|_{*}\left\|f_{1}\right\|_{M \dot{K}_{q_{1}, p_{1}(\cdot)}^{\alpha_{1}\left(\cdot, \lambda_{1}\left(\mathbb{R}^{n}\right)\right.}},
$$


so we only compute $I_{32}(L)$. From inequality (19) and $n \delta_{1}+$ $\alpha_{2}(0)-\lambda_{2}>0$, we obtain

$I_{32}(L)$

$=2^{-L \lambda_{2}}\left\|b_{2}\right\|_{*}\left\{\sum_{k=-\infty}^{L}\left(\sum_{l_{2}=k+2}^{\infty}\left(l_{2}-k\right) 2^{\left(k-l_{2}\right)\left(n \delta_{1}+\alpha_{2}(0)\right)} 2^{l_{2} \alpha_{2}(0)}\right.\right.$

$$
\left.\left.\times\left\|f_{2} \chi_{l_{2}}\right\|_{L^{p_{2}(\cdot)\left(\mathbb{R}^{n}\right)}}\right)^{q_{2}}\right\}^{1 / q_{2}}
$$

$\leq C 2^{-L \lambda_{2}}\left\|b_{2}\right\|_{*}$

$\times\left\{\sum_{k=-\infty}^{L}\left[\sum_{l_{2}=k+2}^{\infty}\left(l_{2}-k\right) 2^{\left(k-l_{2}\right)\left(n \delta_{1}+\alpha_{2}(0)\right)} 2^{l_{2} \lambda_{2}}\right.\right.$

$$
\times 2^{-l_{2} \lambda_{2}}\left(\sum_{m=-\infty}^{l_{2}} 2^{m \alpha_{2}(0) q_{2}}\right.
$$

$\leq C 2^{-L \lambda_{2}}\left\|b_{2}\right\|_{*}\left\{\sum_{k=-\infty}^{L} 2^{k \lambda_{2} q_{2}}\right.$

$$
\left.\left.\left.\times\left\|f_{2} \chi_{m}\right\|_{L^{p_{2}(\cdot)}\left(\mathbb{R}^{n}\right)}^{q_{2}}\right)^{1 / q_{2}}\right]^{q_{2}}\right\}^{1 / q_{2}}
$$

$$
\times\left(\sum_{l_{2}=k+2}^{\infty}\left(l_{2}-k\right) 2^{\left(k-l_{2}\right)\left(n \delta_{1}+\alpha_{2}(0)-\lambda_{2}\right)}\right.
$$

$$
\left.\left.\times\left\|f_{2}\right\|_{M \dot{K}_{q_{2}, p_{2}(\cdot)}^{\alpha_{2}(\cdot), \lambda_{2}\left(\mathbb{R}^{n}\right)}}\right)^{q_{2}}\right\}^{1 / q_{2}}
$$

$\leq C\left\|b_{2}\right\|_{*} 2^{-L \lambda_{2}}\left(\sum_{k=-\infty}^{L} 2^{k \lambda_{2} q_{2}}\right)^{1 / q_{2}}\left\|f_{2}\right\|_{M \dot{K}_{\alpha_{2}, p_{2}(\cdot)}^{\alpha_{2}\left(\cdot, \lambda_{2}\left(\mathbb{R}^{n}\right)\right.}}$

$\leq C\left\|b_{2}\right\|_{*}\left\|f_{2}\right\|_{M \dot{K}_{q_{2} \cdot p_{2} \cdot(\cdot)}^{\alpha_{2}(\cdot), \lambda_{2}}\left(\mathbb{R}^{n}\right)}$.

Therefore,

$$
\begin{aligned}
I_{3} & \leq C \sup _{L \leq 0, L \in \mathbb{Z}} I_{31}(L) I_{32}(L) \\
& \leq C\left\|b_{1}\right\|_{*}\left\|b_{2}\right\|_{*}\left\|f_{1}\right\|_{M \dot{K}_{q_{1}, p_{1}(\cdot)}^{\alpha_{1}\left(\cdot, \lambda_{1}\right.}\left(\mathbb{R}^{n}\right)}\left\|f_{2}\right\|_{M \dot{K}_{q_{2} \cdot p_{2}(\cdot)}^{\alpha_{2}(\cdot), \lambda_{2}}\left(\mathbb{R}^{n}\right)} .
\end{aligned}
$$

Step 4. It turns to estimate the term $I_{5}$. Applying the Hölder inequality and Lemma 13, we have

$$
\begin{aligned}
I_{5} \leq C & \sup _{L \leq 0, L \in \mathbb{Z}} 2^{-L \lambda} \\
& \times\left\{\sum _ { k = - \infty } ^ { L } 2 ^ { k \alpha ( 0 ) q } \left(\sum_{l_{1}=k-1}^{k+1} \sum_{l_{2}=k-1}^{k+1} \|\left[b_{1}, b_{2}, T\right]\right.\right. \\
& \left.\left.\times\left(f_{l_{1}}, f_{l_{2}}\right) \|_{\left.L^{p \cdot(}\right)\left(\mathbb{R}^{n}\right)}\right)^{q}\right\}^{1 / q}
\end{aligned}
$$

$$
\leq C \sup _{L \leq 0, L \in \mathbb{Z}} 2^{-L \lambda}\left\{\sum_{k=-\infty}^{L} 2^{k \alpha(0) q}\right.
$$$$
\times\left(\sum_{l_{1}=k-1}^{k+1} \sum_{l_{2}=k-1}^{k+1}\left\|b_{1}\right\|_{*}\left\|b_{2}\right\|_{*}\right.
$$$$
\left.\left.\times\left\|f_{l_{1}}\right\|_{L^{p_{1} \cdot(\cdot)}\left(\mathbb{R}^{n}\right)}\left\|f_{l_{2}}\right\|_{L^{p_{2} \cdot(\cdot)\left(\mathbb{R}^{n}\right)}}\right)^{q}\right\}^{1 / q}
$$$$
\leq C \prod_{i=1}^{2}\left\|b_{i}\right\|_{*} \sup _{L \leq 0, L \in \mathbb{Z}} 2^{-L \lambda_{i}}
$$$$
\times\left\{\sum_{k=-\infty}^{L} 2^{k \alpha_{i}(0) q_{i}}\left(\sum_{l_{i}=k-1}^{k+1}\left\|f_{l_{i}}\right\|_{L^{p_{i}(\cdot)}\left(\mathbb{R}^{n}\right)}\right)^{q_{i}}\right\}^{1 / q_{i}}
$$$$
\leq C \prod_{i=1}^{2}\left\|b_{i}\right\|_{*} \sup _{L \leq 0, L \in \mathbb{Z}} 2^{-L \lambda_{i}}
$$$$
\times\left(\sum_{k=-\infty}^{L} 2^{k \alpha_{i}(0) q_{i}}\left\|f_{i} \chi_{k}\right\|_{L^{p_{i}(\cdot)\left(\mathbb{R}^{n}\right)}}^{q_{i}}\right)^{1 / q_{i}}
$$$$
\leq C\left\|b_{1}\right\|_{*}\left\|b_{2}\right\|_{*}\left\|f_{1}\right\|_{M \dot{K}_{q_{1}, p_{1} \cdot(\cdot)}^{\alpha_{1}\left(\cdot, \lambda_{1}\right.}\left(\mathbb{R}^{n}\right)}
$$$$
\times\left\|f_{2}\right\|_{M \dot{K}_{q_{2}, p_{2}(\cdot)}^{\alpha_{2}(\cdot), \lambda_{2}\left(\mathbb{R}^{n}\right)}} .
$$

Step 5. Now it goes to the estimate of $I_{6}$.

It is clear that, for $x \in D_{k}$ and $k-1 \leq l_{1} \leq k+1$ and $l_{2} \geq k+2$,

$$
\begin{aligned}
& \left|\left[b_{1}, b_{2}, T\right]\left(f_{1}, f_{2}\right)(x)\right| \\
& \leq C 2^{-k n} \int_{\mathbb{R}^{n}}\left[b_{1}(x)-b_{1}\left(y_{1}\right)\right] f_{1}\left(y_{1}\right) d y_{1} 2^{-l_{2} n} \\
& \quad \times \int_{\mathbb{R}^{n}}\left[b_{2}(x)-b_{2}\left(y_{2}\right)\right] f_{2}\left(y_{2}\right) d y_{2} .
\end{aligned}
$$

Therefore,

$$
\begin{aligned}
& \left\|\sum_{l_{1}=k-1}^{k+1} \sum_{l_{2}=k+2}^{\infty}\left[b_{1}, b_{2}, T\right]\left(f_{l_{1}}, f_{l_{2}}\right) \chi_{k}\right\|_{L^{p^{(\cdot)}\left(\mathbb{R}^{n}\right)}} \\
& \leq C\left\|b_{1}\right\|_{*}\left\|b_{2}\right\|_{*} \\
& \quad \times \sum_{l_{1}=k-1}^{k+1} \sum_{l_{2}=k+2}^{\infty} 2^{\left(l_{1}-k\right) n}\left\|f_{l_{1}}\right\|_{L^{p_{1}(\cdot)}\left(\mathbb{R}^{n}\right)} \\
& \quad \times\left(l_{2}-k\right) 2^{\left(k-l_{2}\right) n \delta_{1}}\left\|b_{2}\right\|_{*}\left\|f_{l_{2}}\right\|_{L^{p_{2}(\cdot)}\left(\mathbb{R}^{n}\right)^{\prime}},
\end{aligned}
$$




$$
\begin{aligned}
& I_{6} \leq C \sup _{L \leq 0, L \in \mathbb{Z}} 2^{-L \lambda} \\
& \times\left\{\sum _ { k = - \infty } ^ { L } 2 ^ { k \alpha ( 0 ) q } \left(\sum_{l_{1}=k-1}^{k+1} \sum_{l_{2}=k+2}^{\infty} \|\left[b_{1}, b_{2}, T\right]\right.\right. \\
& \left.\left.\times\left(f_{l_{1}}, f_{l_{2}}\right) \|_{L^{p(\cdot)}\left(\mathbb{R}^{n}\right)}\right)^{q}\right\}^{1 / q} \\
& \leq C \sup _{L \leq 0, L \in \mathbb{Z}} 2^{-L \lambda}\left\|b_{1}\right\|_{*}\left\|b_{2}\right\|_{*} \\
& \times\left\{\sum _ { k = - \infty } ^ { L } 2 ^ { k \alpha ( 0 ) q } \left(\sum_{l_{1}=k-1}^{k+1} \sum_{l_{2}=k+2}^{\infty} 2^{\left(l_{1}-k\right) n}\left\|f_{l_{1}}\right\|_{L^{p_{1}(\cdot)}\left(\mathbb{R}^{n}\right)}\right.\right. \\
& \left.\left.\times\left(l_{2}-k\right) 2^{\left(k-l_{2}\right) n \delta_{1}}\left\|f_{l_{2}}\right\|_{L^{p_{2}(\cdot)}\left(\mathbb{R}^{n}\right)}\right)^{q}\right\}^{1 / q} \\
& \leq C \sup _{L \leq 0, L \in \mathbb{Z}} 2^{-L \lambda_{1}}\left\|b_{1}\right\|_{*} \\
& \times\left\{\sum_{k=-\infty}^{L}\left(\sum_{l_{1}=k-1}^{k+1} 2^{k \alpha_{1}(0)} 2^{\left(l_{1}-k\right) n}\left\|f_{l_{1}}\right\|_{L^{p_{1}(\cdot)}\left(\mathbb{R}^{n}\right)}\right)^{q_{1}}\right\}^{1 / q_{1}} \\
& \times 2^{-L \lambda_{2}}\left\|b_{2}\right\|_{*}\left\{\sum _ { k = - \infty } ^ { L } \left(\sum_{l_{2}=k+2}^{\infty}\left(l_{2}-k\right) 2^{\left(k-l_{2}\right)\left(n \delta_{1}+\alpha_{2}(0)\right)}\right.\right. \\
& \left.\left.\times 2^{l_{2} \alpha_{2}(0)}\left\|f_{l_{2}}\right\|_{L^{p_{2}(\cdot)}\left(\mathbb{R}^{n}\right)}\right)^{q_{2}}\right\}^{1 / q_{2}} \\
& =: C \sup _{L \leq 0, L \in \mathbb{Z}} I_{61}(L) I_{62}(L) \text {. }
\end{aligned}
$$

Here, the estimate of $I_{61}(L)$ is similar to that of $I_{22}(L)$ and $I_{62}(L)=I_{32}(L)$.

Step 6. Finally, we will finish the estimation of the last term $I_{9}$. Note that $l_{2} \geq k+2$ and $\left|x-y_{i}\right|>2^{l_{i}-2}$ for $x \in D_{k}, y_{i} \in D_{l_{i}}$, $i=1,2$ : we get

$$
\begin{aligned}
& {\left[b_{1}, b_{2}, T\right]\left(f_{l_{1}}, f_{l_{2}}\right)(x)} \\
& \leq C 2^{-l_{1} n} \int_{\mathbb{R}^{n}}\left[b_{1}(x)-b_{1}\left(y_{1}\right)\right] f_{l_{1}}\left(y_{1}\right) d y_{1} \cdot 2^{-l_{2} n} \\
& \quad \times \int_{\mathbb{R}^{n}}\left[b_{2}(x)-b_{2}\left(y_{2}\right)\right] f_{l_{2}}\left(y_{2}\right) d y_{2} .
\end{aligned}
$$

Applying the Hölder inequality to the last integral, we obtain

$$
\begin{aligned}
& \left\|\sum_{l_{1}=k+2}^{\infty} \sum_{l_{2}=k+2}^{\infty}\left[b_{1}, b_{2}, T\right]\left(f_{l_{1}}, f_{l_{2}}\right) \chi_{k}\right\|_{L^{p(\cdot)}\left(\mathbb{R}^{n}\right)} \\
& \quad \leq C\left\|b_{1}\right\|_{*}\left\|b_{2}\right\|_{*}
\end{aligned}
$$

$$
\begin{aligned}
& \times \sum_{l_{1}=k+2 l_{2}=k+2}^{\infty} \sum^{\infty}\left(l_{1}-k\right) 2^{\left(k-l_{1}\right) n \delta_{1}}\left\|f_{l_{1}}\right\|_{L^{p_{1}(\cdot)}\left(\mathbb{R}^{n}\right)} \\
& \times\left(l_{2}-k\right) 2^{\left(k-l_{2}\right) n \delta_{1}}\left\|f_{l_{2}}\right\|_{L^{p_{2}(\cdot)}\left(\mathbb{R}^{n}\right)} \\
\leq & C \prod_{i=1}^{2}\left\|b_{i}\right\|_{*} \sum_{l_{i}=k+2}^{\infty}\left(l_{i}-k\right) 2^{\left(k-l_{i}\right) n \delta_{1}}\left\|f_{l_{i}}\right\|_{L^{p_{i}(\cdot)}\left(\mathbb{R}^{n}\right)} .
\end{aligned}
$$

Thus,

$$
\begin{aligned}
& I_{9} \leq C \sup _{L \leq 0, L \in \mathbb{Z}} 2^{-L \lambda} \\
& \times\left\{\sum _ { k = - \infty } ^ { L } 2 ^ { k \alpha ( 0 ) q } \left(\sum_{l_{1}=k+2}^{\infty} \sum_{l_{2}=k+2}^{\infty} \|\left[b_{1}, b_{2}, T\right]\right.\right. \\
& \left.\left.\times\left(f_{l_{1}}, f_{l_{2}}\right) \|_{L^{p(\cdot)}\left(\mathbb{R}^{n}\right)}\right)^{q}\right\}^{1 / q} \\
& \leq C \sup _{L \leq 0, L \in \mathbb{Z}} 2^{-L \lambda} \\
& \times\left\{\sum _ { k = - \infty } ^ { L } 2 ^ { k \alpha ( 0 ) q } \left(\prod_{i=1}^{2}\left\|b_{i}\right\|_{*} \sum_{l_{i}=k+2}^{\infty}\left(l_{i}-k\right)\right.\right. \\
& \left.\left.\times 2^{\left(k-l_{i}\right) n \delta_{1}}\left\|f_{l_{i}}\right\|_{L^{p_{i}(\cdot)}\left(\mathbb{R}^{n}\right)}\right)^{q}\right\}^{1 / q} \\
& \leq C \sup _{L \leq 0, L \in \mathbb{Z}} \prod_{i=1}^{2} 2^{-L \lambda_{i}}\left\|b_{i}\right\|_{*} \\
& \times\left\{\sum _ { k = - \infty } ^ { L } \left(\sum_{l_{i}=k+2}^{\infty}\left(l_{i}-k\right) 2^{\left(k-l_{i}\right)\left(n \delta_{1}+\alpha_{i}(0)\right)}\right.\right. \\
& \left.\left.\times 2^{l_{i} \alpha_{i}(0)}\left\|f_{l_{i}}\right\|_{L^{p^{i}(\cdot)}\left(\mathbb{R}^{n}\right)}\right)^{q_{i}}\right\}^{1 / q_{i}} \\
& =: C \sup _{L \leq 0, L \in \mathbb{Z}} I_{91}(L) I_{92}(L) \\
& \leq C\left\|b_{1}\right\|_{*}\left\|b_{2}\right\|_{*}\left\|f_{1}\right\|_{M \dot{K}_{q_{1}, p_{1}(\cdot)}^{\alpha_{1}(\cdot), \lambda_{1}}\left(\mathbb{R}^{n}\right)} \\
& \times\left\|f_{2}\right\|_{M \dot{K}_{q_{2}, p_{2}(\cdot)}^{\alpha_{2}(\cdot), \lambda_{2}}\left(\mathbb{R}^{n}\right)^{*}} .
\end{aligned}
$$

Here, the estimate of $I_{9 i}(L), i=1,2$, is similar to that of $I_{32}(L)$.

Combing all estimates for $I_{i}, i=1,2, \ldots, 9$ together, we get

$E \leq C\left\|b_{1}\right\|_{*}\left\|b_{2}\right\|_{*}\left\|f_{1}\right\|_{M \dot{K}_{q_{1}, p_{1}(\cdot)}^{\alpha_{1}(\cdot), \lambda_{1}}\left(\mathbb{R}^{n}\right)}\left\|f_{2}\right\|_{M \dot{K}_{q_{2}, p_{2}(\cdot)}^{\alpha_{2}(\cdot), \lambda_{2}}\left(\mathbb{R}^{n}\right)}$.

This finishes the proof of Theorem 6 . 


\section{Conflict of Interests}

The authors declare that there is no conflict of interests regarding the publication of this paper.

\section{Acknowledgments}

The authors would like to thank the referee for his suggestion. The first author was supported by the National Natural Science Foundation of China (Grant no. 11021043) and the Fundamental Research Funds for the Central Universities. The third author was supported by the National Natural Science Foundation of China (Grants nos. 11071064 and 11361020) and the Natural Science Foundation of Hainan Province (no. 113004).

\section{References}

[1] O. Kováčik and J. Rákosník, "On spaces $L^{p(x)}$ and $W^{k, p(x), "}$ Czechoslovak Mathematical Journal, vol. 41, no. 4, pp. 592-618, 1991.

[2] A. Almeida and D. Drihem, "Maximal, potential and singular type operators on Herz spaces with variable exponents," Journal of Mathematical Analysis and Applications, vol. 394, no. 2, pp. 781-795, 2012.

[3] A. Almeida, J. Hasanov, and S. Samko, "Maximal and potential operators in variable exponent Morrey spaces," Georgian Mathematical Journal, vol. 15, no. 2, pp. 195-208, 2008.

[4] A. Almeida and P. Hästö, "Besov spaces with variable smoothness and integrability," Journal of Functional Analysis, vol. 258, no. 5, pp. 1628-1655, 2010.

[5] L. Diening, P. Hästö, and S. Roudenko, "Function spaces of variable smoothness and integrability," Journal of Functional Analysis, vol. 256, no. 6, pp. 1731-1768, 2009.

[6] B. H. Dong and J. S. Xu, "New Herz type Besov and TriebelLizorkin spaces with variable exponents," Journal of Function Spaces and Applications, vol. 2012, Article ID 384593, 27 pages, 2012.

[7] P. Gurka, P. Harjulehto, and A. Nekvinda, "Bessel potential spaces with variable exponent," Mathematical Inequalities \& Applications, vol. 10, no. 3, pp. 661-676, 2007.

[8] M. Izuki, "Boundedness of vector-valued sublinear operators on Herz-Morrey spaces with variable exponent," Mathematical Sciences Research Journal, vol. 13, no. 10, pp. 243-253, 2009.

[9] H. Kempka, "2-microlocal Besov and Triebel-Lizorkin spaces of variable integrability," Revista Matemática Complutense, vol. 22, no. 1, pp. 227-251, 2009.

[10] H. Kempka, "Atomic, molecular and wavelet decomposition of generalized 2-microlocal Besov spaces," Journal of Function Spaces and Applications, vol. 8, no. 2, pp. 129-165, 2010.

[11] E. Nakai and Y. Sawano, "Hardy spaces with variable exponents and generalized Campanato spaces," Journal of Functional Analysis, vol. 262, no. 9, pp. 3665-3748, 2012.

[12] Y. Sawano, "Atomic decompositions of Hardy spaces with variable exponents and its application to bounded linear operators," Integral Equations and Operator Theory, vol. 77, no. 1, pp. 123148, 2013.
[13] H. Wang and Z. Liu, "The Herz-type Hardy spaces with variable exponent and their applications," Taiwanese Journal of Mathematics, vol. 16, no. 4, pp. 1363-1389, 2012.

[14] A.-W. Huang and J.-S. Xu, "Multilinear singular integrals and commutators in variable exponent Lebesgue spaces," Applied Mathematics-A Journal of Chinese Universities, vol. 25, no. 1, pp. 69-77, 2010.

[15] X. Tao and H. Zhang, "On the boundedness of multilinear operators on weighted Herz-Morrey spaces," Taiwanese Journal of Mathematics, vol. 15, no. 4, pp. 1527-1543, 2011.

[16] W. Wang and J. Xu, "Commutators of multilinear singular integrals with Lipschitz functions on products of variable exponent Lebesgue spaces," Advances in Mathematics, vol. 38, no. 6, pp. 669-677, 2009 (Chinese).

[17] W. Wang and J.-s. Xu, "Commutators of multilinear singular integrals with Lipschitz functions," Communications in Mathematical Research, vol. 25, no. 4, pp. 318-328, 2009.

[18] J. Xu, "Generalized commutators of multilinear singular integrals," Proceedings of A. Razmadze Mathematical Institute, vol. 142, pp. 109-122, 2006.

[19] J. S. Xu, "Multilinear commutators of multilinear singular integrals," Acta Mathematica Sinica, vol. 51, no. 5, pp. 1021-1034, 2008.

[20] X. T. Duong, L. Grafakos, and L. Yan, "Multilinear operators with non-smooth kernels and commutators of singular integrals," Transactions of the American Mathematical Society, vol. 362, no. 4, pp. 2089-2113, 2010.

[21] J.-1. Lian, J. Li, and H.-x. Wu, "Multilinear commutators of BMO functions and multilinear singular integrals with nonsmooth kernels," Applied Mathematics-A Journal of Chinese Universities, vol. 26, no. 1, pp. 109-120, 2011.

[22] Z. H. Xuan and L. S. Shu, "Boundedness of higher order commutators on Herz-Morrey spaces with variable exponent," Journal of Nanjing University Mathematical Biquarterly, vol. 30, no. 2, pp. 188-196, 2013.

[23] D. X. Chen and S. Z. Mao, "Weighted estimates for maximal commutators of multilinear singular integrals," Journal of Function Spaces and Applications, vol. 2012, Article ID 128520, 20 pages, 2012.

[24] L. Tang, "Weighted estimates for vector-valued commutators of multilinear operators," Proceedings of the Royal Society of Edinburgh A, vol. 138, no. 4, pp. 897-922, 2008.

[25] L. Grafakos and R. H. Torres, "Multilinear Calderón-Zygmund theory," Advances in Mathematics, vol. 165, no. 1, pp. 124-164, 2002.

[26] L. Grafakos and R. H. Torres, "Maximal operator and weighted norm inequalities for multilinear singular integrals," Indiana University Mathematics Journal, vol. 51, no. 5, pp. 1261-1276, 2002.

[27] D. V. Cruz-Uribe and A. Fiorenza, Variable Lebesgue Spaces: Foundations and Harmonic Analysis, Applied and Numerical Harmonic Analysis, Springer, Heidelberg, Germany, 2013.

[28] L. Diening, P. Harjulehto, P. Hästö, and M. Ružička, Lebesgue and Sobolev Spaces with Variable Exponents, vol. 2017 of Lecture Notes in Mathematics, Springer, Berlin, Germany, 2011.

[29] M. Izuki, "Boundedness of commutators on Herz spaces with variable exponent," Rendiconti del Circolo Matematico di Palermo, vol. 59, no. 2, pp. 199-213, 2010. 
[30] M. Izuki, "Fractional integrals on Herz-Morrey spaces with variable exponent," Hiroshima Mathematical Journal, vol. 40, no. 3, pp. 343-355, 2010.

[31] S. Z. Lu, D. C. Yang, and G. Hu, Herz Type Spaces and their Applications, Science Press, Bejing, China, 2008.

[32] Z. H. Xuan and L. S. Shu, "Vector-estimate of higher order commutators on Herz-Morrey spaces with variable exponent," Acta Mathematica Sinica. In press. 


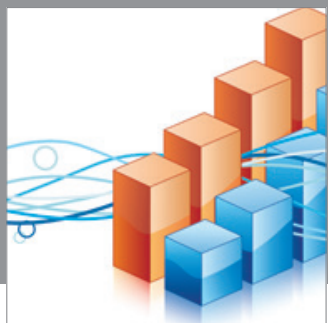

Advances in

Operations Research

mansans

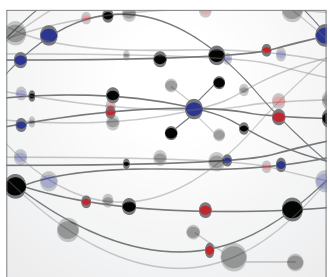

The Scientific World Journal
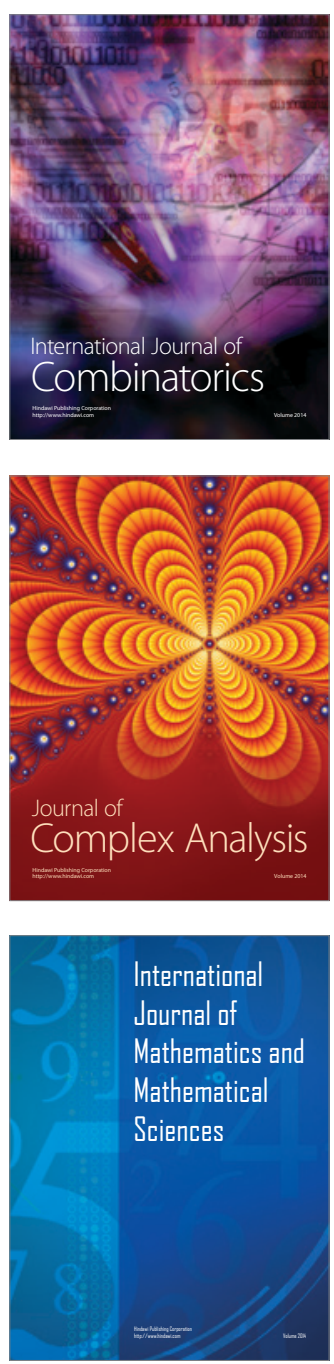
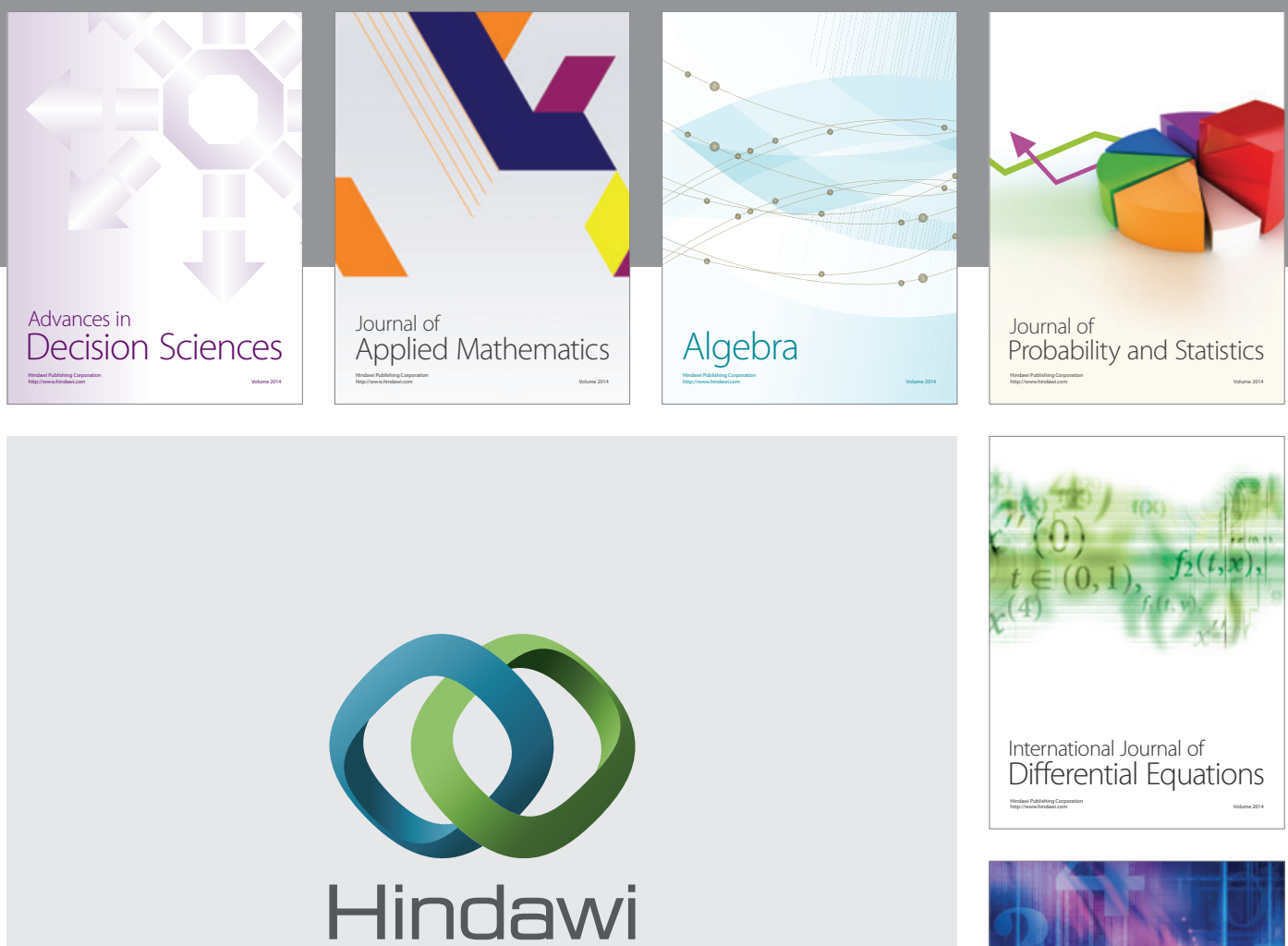

Submit your manuscripts at http://www.hindawi.com
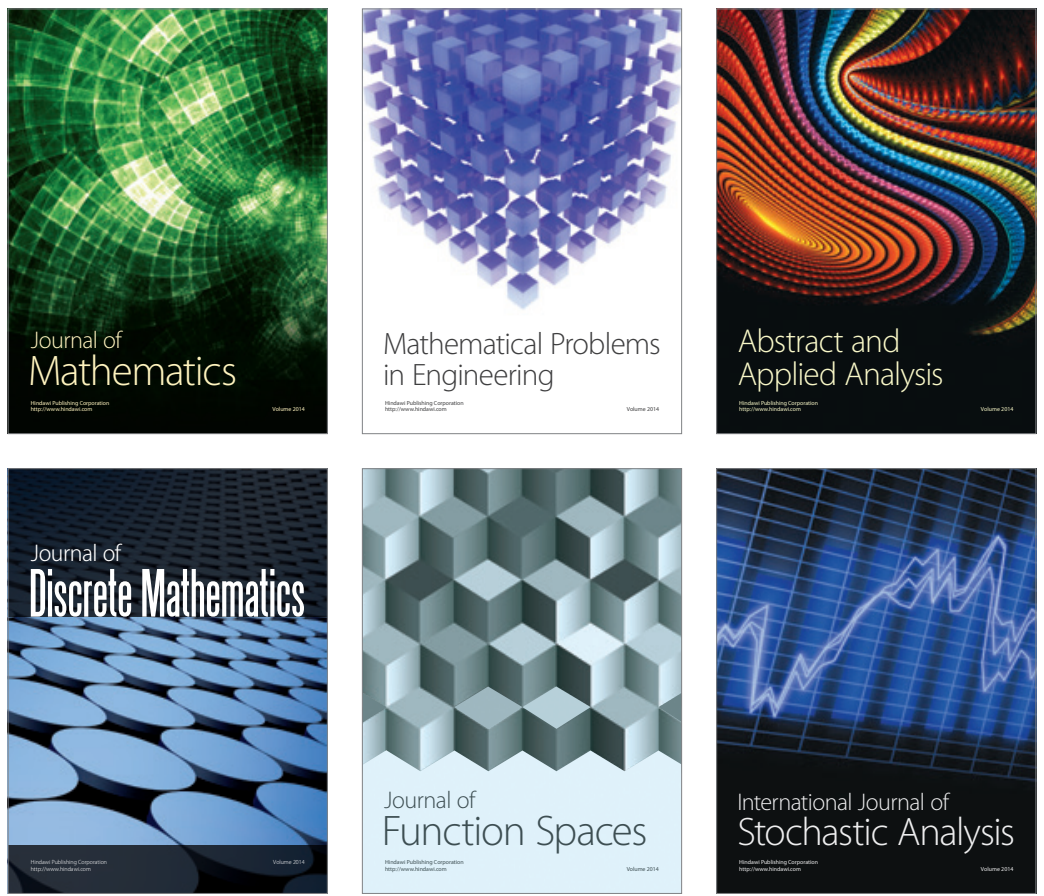

Journal of

Function Spaces

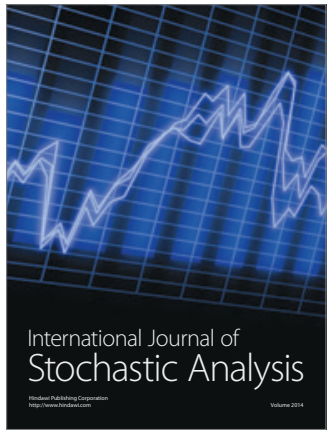

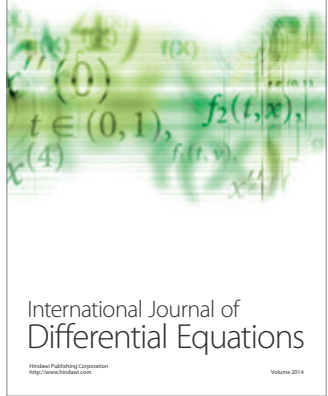
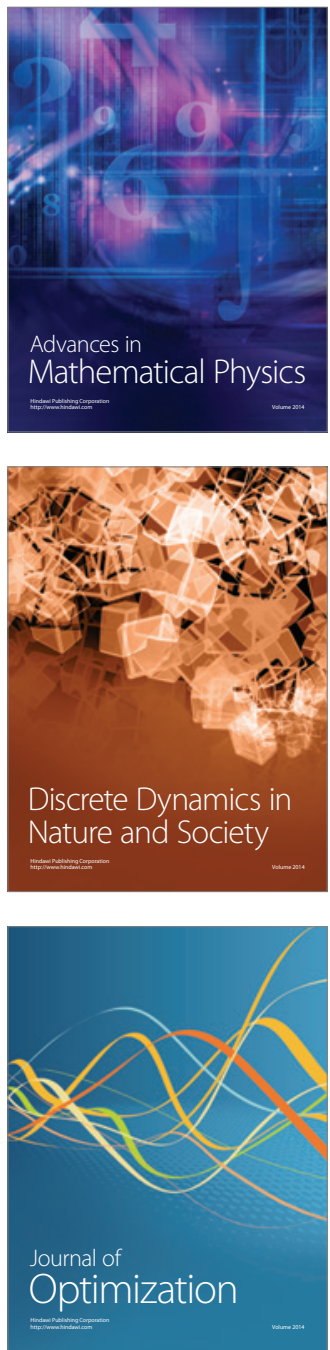